HIGOR LANDGRAF

AVALIAÇÃO DA ESTABILIDADE NA FIXAÇÃO DA OSTEOTOMIA SAGITAL MANDIBULAR UTILIZANDO PARAFUSOS BICORTICAIS INSERIDOS COM ANGULAÇÃO DE $60^{\circ} \mathrm{E} 90^{\circ}$. ESTUDO BIOMECÂNICO EM MANDÍBULAS DE CARNEIRO 


\section{Higor Landgraf}

\section{Avaliação da estabilidade na fixação da osteotomia sagital mandibular utilizando parafusos bicorticais inseridos com angulação de $60^{\circ}$ e $90^{\circ}$. Estudo biomecânico em mandíbulas de carneiro}

Dissertação apresentada à Faculdade de Odontologia da Universidade de São Paulo, para obter o título de Mestre pelo Programa de Pós-Graduação em Ciências Odontológicas.

Área de Concentração: Cirurgia e Traumatologia Buco-Maxilo-Faciais

Orientador: Prof. Dr. Elio Hitoshi Shinohara 
Catalogação-na-Publicação

Serviço de Documentação Odontológica

Faculdade de Odontologia da Universidade de São Paulo

Landgraf, Higor

Avaliação da estabilidade na fixação da osteotomia sagital mandibular utilizando parafusos bicorticais inseridos com angulação de $60^{\circ}$ e $90^{\circ}$. Estudo biomecânico em mandíbulas de carneiro / Higor Landgraf; orientador Elio Hitoshi Shinohara. -- São Paulo, 2008.

67p.: tab., fig.; $30 \mathrm{~cm}$.

Dissertação (Mestrado - Programa de Pós-Graduação em Odontologia. Área de Concentração: Cirurgia e Traumatologia Buco-Maxilo-Faciais) -- Faculdade de Odontologia da Universidade de São Paulo.

1. Osteotomia sagital mandibular - Parafusos bicorticais - Angulação Mandíbula - Osteotomia - Biomecânica 3. Cirurgia bucomaxilofacial

CDD 617.605

BLACK D721

AUTORIZO A REPRODUÇÃO E DIVULGAÇÃO TOTAL OU PARCIAL DESTE TRABALHO, POR QUALQUER MEIO CONVENCIONAL OU ELETRÔNICO, PARA FINS DE ESTUDO E PESQUISA, DESDE QUE CITADA A FONTE E COMUNICADA AO AUTOR A REFERÊNCIA DA CITAÇÃO.

São Paulo,

Assinatura:

E-mail:higorland@usp.br 
Landgraf H. Avaliação da estabilidade na fixação da osteotomia sagital mandibular utilizando parafusos bicorticais inseridos com angulação de $60^{\circ} \mathrm{e} 90^{\circ}$. Estudo biomecânico em mandíbulas de carneiro [Dissertação de Mestrado]. São Paulo: Faculdade de Odontologia da USP; 2008.

São Paulo, 25/11/2008

\section{Banca Examinadora}

1) $\operatorname{Prof}(a)$. $\operatorname{Dr}(a)$.

Titulação:

Julgamento:

Assinatura:

2) $\operatorname{Prof}(a) \cdot \operatorname{Dr}(a)$

Titulação:

Julgamento:

Assinatura:

3) $\operatorname{Prof}(a)$. $\operatorname{Dr}(a)$.

Titulação:

Julgamento: Assinatura: 


\section{DEDICATÓRIA}

A Deus, meu maior amigo, que sempre guiou meus passos e me orienta nos momentos difíceis.

Aos meus pais, Hugo José Landgraf e Marlene Vagacz Landgraf, pela dedicação, educação, carinho, incentivo e compreensão que sempre tiveram. Meus maiores exemplos na vida.

Aos meus irmãos e grandes amigos, Hugo José Landgraf Júnior e Horst Landgraf, pelo companheirismo e incentivo que sempre me demonstraram.

Ao meu amor, Larissa Klosovski Schon, que cruzou em minha vida durante a pósgraduação, pelo carinho, saudade e compreensão da minha ausência e distância. 


\section{AGRADECIMENTO ESPECIAL}

Ao Prof. Dr. Elio Hitoshi Shinohara, meu orientador, amigo, que sempre acreditou, me apoiou e incentivou, entendendo meus erros e problemas. Mesmo que ainda desconhecidos na época, quando ainda cursava a graduação e estava preparando uma apresentação, sentou ao meu lado ajudando-me nesta, da mesma forma que se dispôs e sempre me ajudou em outras ocasiões, principalmente durante meu estágio hospitalar. Pessoa esta que comecei a admirar, seguindo seus passos e que se tornou minha referência devido ao "grande profissional e grande ser humano" que representa. Após o término de minha residência, tive o prazer de reencontrá-lo na pós-graduação, agora não como professor e amigo, mas sim como orientador e amigo. Meus sinceros agradecimentos. 


\section{AGRADECIMENTOS}

À Universidade de São Paulo - USP, através da Faculdade de Odontologia FOUSP, pela estrutura e oportunidade para a formação profissional e docente e ser instituição de referência para todas as áreas do conhecimento.

À Universidade Estadual Paulista - UNESP, através da Faculdade de Odontologia de Araraquara FOAr-UNESP, pela oportunidade e formação profissional e ser instituição de referência nacional na área de cirurgia e traumatologia bucomaxilofacial.

Ao Conjunto Hospitalar do Mandaqui - SUS - SP, pela oportunidade de estagiar e preparo para ingressar na residência em CTBMF.

À Universidade de Marília, através da Faculdade de Odontologia, pela minha formação como Cirurgião Dentista.

Ao Departamento de Cirurgia, Prótese e Traumatologia Buco-Maxilo-Faciais da Faculdade de Odontologia da Universidade de São Paulo, pela oportunidade e estrutura disponível.

Ao Departamento de Biomateriais e Bioquímica Oral da Faculdade de Odontologia da Universidade de São Paulo, pela possibilidade e utilização da máquina Instron, utilização dos laboratórios e realização do experimento. 
Aos professores da Área de Cirurgia e Traumatologia Buco-Maxilo-Facial da Faculdade de Odontologia da Universidade de São Paulo.

Ao Prof. Titular Dr. Rafael Yagüe Ballester, pelo aprendizado, pela orientação e empenho durante a parte experimental do trabalho, pela seriedade, competência e dedicação que conduz a pós-graduação.

Ao Prof. Dr. Antonio Muench pela ajuda na análise e interpretação estatística.

Aos meus colegas de mestrado, Júlio Mancini, Luis Augusto Vanti e Luis Felipe Nakamai, pessoas com quem muito aprendi, pela grande amizade, pelo respeito mútuo e pela oportunidade de conviver com pessoas de grande valor.

Aos demais colegas do curso de pós-graduação.

À colega de pós-graduação Tathy Aparecida Xavier, pela amizade, ajuda e auxílio na execução do ensaio mecânico.

À Prof. ${ }^{a}$ Dra. Fabiana Soares Grecca e Prof. Expedito Machado de Faria, pelo apoio, incentivo e orientação nas apresentações em jornadas acadêmicas, durante período da graduação.

Ao Prof. Dr. José Sidney Roque, pela seriedade e competência que conduz a docência, pela oportunidade de estagiar e monitorar nas disciplinas de anatomia e cirurgia, durante o período de graduação. 
Ao colega e amigo José Pereira de Carvalho Júnior, pela amizade, aprendizado, conselhos e simplicidade do grande cirurgião que representa.

Ao colega e amigo Prof. Dr. Ricardo Curcio, pela amizade, aprendizado e oportunidade profissional.

Ao colega Felipe Ladeira Pereira, pela amizade e convívio durante período de estágio hospitalar, pessoa com quem muito aprendi, pelo crescimento conjunto e amizade formada.

Às colegas Silvia Maria Marques D'Andretta, Salete Maria Davenis e Thais Annunciato pelo aprendizado e convívio durante período de estágio hospitalar.

Aos professores da Área de Cirurgia e Traumatologia Buco-Maxilo-Facial da Faculdade de Odontologia da Universidade Estadual Paulista UNESP, campos de Araraquara FOAr -UNESP, Prof. Titular Dr. Mario Francisco Real Gabrielli, Profa. Dra. Marisa Aparecida Cabrini Gabrielli, Prof. Dr. Eduardo Hochuli Vieira, Prof. Dr. Valfrido Antonio Pereira Filho, Prof. Dr. Roberto Henrique Barbeiro e Prof. Dr. José Scarso Filho, pelo aprendizado, sólida formação profissional, amizade, pela competência e dedicação com que conduzem a CTBMF e a formação dos novos cirurgiões.

Aos colegas de residência, Marcelo Silva Monnazzi, Daniela Prata, Leandro Benetti de Oliveira, Daniel Bittencourt Schimidt, Leandro Eduardo Kluppel, Adriano Freitas de Assis, Elis Cristina Souza Serra, João Frank Dantas e 
Guilherme Romano Scartezini, com os quais convivi e muito aprendi, pela grande amizade, respeito e oportunidade de conhecer pessoas de grande valor.

Ao Prof. Dr. Fabio Petroucic, pela confiança e oportunidade profissional.

Ao Prof. Dr. Sergio Luis de Miranda, pela amizade, aprendizado, confiança e oportunidade profissional.

Aos colegas Bruna Fontes e Roberto Moreno, pela amizade, convívio e aprendizado.

À Thelma Aparecida Gomes e Sra Juraci, pela amizade, convivência e carinho dispensados.

Ao Sr. Silvio, pelo auxílio laboratorial na execução do experimento.

À minha tia Sonia e família, pela forma tão especial que sempre me acolheram em sua casa.

A todos que contribuíram de alguma forma para a realização deste trabalho. 
"A alegria que se tem em pensar e aprender faz-nos pensar e aprender ainda mais."

\title{
William Shakespeare
}

"Grandes realizações não são feitas por impulso, mas por uma soma de pequenas realizações."

Vincent Van Gogh

"Sê dono da tua vontade e escravo da tua consciência."

\author{
Aristóteles
}

"O prazer no trabalho aperfeiçoa a obra."

Aristóteles

"A mente que se abre a uma nova idéia jamais voltará ao seu tamanho original." 
Landgraf $\mathrm{H}$. Avaliação da estabilidade na fixação da osteotomia sagital mandibular utilizando parafusos bicorticais inseridos com angulação de $60^{\circ}$ e $90^{\circ}$. Estudo biomecânico em mandíbulas de carneiro [Dissertação de Mestrado]. São Paulo: Faculdade de Odontologia da USP; 2008.

\section{RESUMO}

Este trabalho foi desenvolvido com a finalidade de verificar a resistência mecânica obtida com parafusos bicorticais posicionais na fixação interna rígida da osteotomia sagital do ramo mandibular, comparando a angulação de inserção dos mesmos. A técnica tradicional e comumente utilizada para fixação da osteotomia sagital mandibular faz uso de parafusos inseridos por meio de acesso extra-oral, com ângulo aproximado de $90^{\circ}$. A principal desvantagem da técnica seria a incisão na pele, tendo potencial de resultar em cicatriz aparente em área visível, bem como risco de lesão à inervação motora da face. Uma alternativa que evita estas complicações é a técnica intra-oral, na qual não há incisão em pele, desta forma sendo bastante vantajosa para o paciente. Nesta, o parafuso é inserido em ângulo próximo de $60^{\circ}$. Visto que, na literatura poucos trabalhos compararam estes dois tipos de fixação, o objetivo deste trabalho foi avaliar a resistência mecânica utilizando parafusos inseridos com angulação de $60^{\circ}$ ou $90^{\circ}$ simulando, respectivamente, as técnicas intra e extra-oral. Foram utilizadas 10 mandíbulas de carneiro, que após receberem osteotomia bilateral do ramo, foram divididas em dois grupos, ambos fixados com parafusos do sistema 2,0mm. Grupo I: fixação em $60^{\circ}$; Grupo II: fixação em $90^{\circ}$. Para o teste mecânico utilizou-se máquina de ensaio universal (Instron 5565), na qual foi acoplado suporte metálico para manter as mandíbulas. Suporte este, que ao contrário dos normalmente utilizados e citados na literatura, permite que a mandíbula seja deformada tridimensionalmente durante o ensaio. Os dados sobre a força foram coletados em Newton e a média calculada com seu desvio padrão para cada grupo. A avaliação estatística dos valores obtidos foi realizada por meio de análise estatística paramétrica. Como resultados, verificamos que o grupo de $90^{\circ}$ apresentou maior resistência mecânica, diferença 
esta que foi estatisticamente significante. Contudo, não houve diferença significante da rigidez entre os dois grupos. Concluímos que o suporte metálico desenvolvido para permitir deformação tridimensional se mostrou eficaz. Acreditamos que desta forma os movimentos mandibulares possam ser melhor reproduzidos durante o ensaio mecânico. Há necessidade de outros estudos visando comparar o sucesso clínico entre as duas técnicas.

Palavras-Chave: Cirurgia Ortognática - Fixação interna rígida - Osteotomia mandibular 
Landgraf $\mathrm{H}$. Evaluation of the stability of mandibular sagital osteotomy fixation using bicortical screws inserted with $60^{\circ}$ and $90^{\circ}$ degrees angles. Biomechanical study in sheep mandibles [Dissertação de Mestrado]. São Paulo: Faculdade de Odontologia da USP; 2008.

\section{ABSTRACT}

This research was developed to evaluate the mechanical resistance obtained with positional bicortical screws used for internal rigid fixation of bicortical split mandibular ramus osteotomy, comparing two different screw insertion angulations. The traditional technique, most commonly used, for the fixation of bicortical split mandibular ramus osteotomy uses approximately $90^{\circ}$ degrees screws inserted through skin incisions. The main disadvantage of this technique may be the skin incision, having the potential of resulting in an apparent scar in a visible area as well as the risk of motor nerve injury. One alternative that avoid these complications is the intra oral technique, in which there's no skin incision, being advantageous to the patient. In this technique the screws are inserted in approximately in $60^{\circ}$ degrees angle. Knowing that in the literature few articles compare these two types of fixation, the objective of this work was to evaluate the mechanical resistance obtained using screws inserted with both $60^{\circ}$ and $90^{\circ}$ degrees angle simulating, respectively, the intra and extra oral techniques. We used 10 sheep mandibles, after performing bicortical sagital split osteotomies, were divided in two groups, both received $2.0 \mathrm{~mm}$ system screws for fixation. Group 1: $60^{\circ}$ fixation; Group 2: $90^{\circ}$ fixation. To realize the mechanical testing a universal rehearsal machine (Instron 5565) was used, in which a metallic support was adapted to restrain the mandibles. This support, differently of the ones commonly used in the current literature, allows that the mandible suffer tridimensional deformation during the rehearsal. The strength data was collected in Newton and the median was calculated with standard deviation for each group. The statistical evaluation of the values obtained was performed using parametrical statistical analysis. Through the results we could verify that the $90^{\circ}$ degrees group presented with statistically significant higher mechanical strength. However, there 
was no significant difference in the rigidity between the two groups. We can conclude that the metallic support developed to allow tridimensional deformation was effective. We believe that by that way mandibular movements can be better reproduced during mechanical rehearsal. There is the necessity of other studies to compare the clinical success between those two fixation techniques.

Key-works: Orthognathic surgery - Internal rigid fixation - Mandibular osteotomy 


\section{LISTA DE ILUSTRAÇÕES}

Figura 4.1 - Vista superior do dispositivo metálico com a mandíbula posicionada....27

Figura 4.2 - Vista súpero-inferior de mandíbulas fixadas com parafusos angulados em $60^{\circ}$ ou $90^{\circ}$, representando o grupo I e II, respectivamente.

Figura 4.3 - Vista frontal da máquina Instron (5565) com a mandíbula posicionada sobre o dispositivo metálico durante o ensaio biomecânico

Figura 4.4 - llustração das três maiores forças atuantes e suas direções sobre a

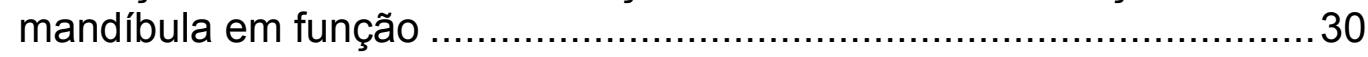

Figura 4.5-Gráfico exemplificando os três ciclos de deslocamento durante a verificação da rigidez 31

Figura 4.6 - Método de cálculo da rigidez 32

Figura 5.1 - Vista medial do ramo mandibular evidenciando falha na fixação após ensaio biomecânico 


\section{LISTA DE TABELAS}

Tabela 5.1 - Análise de variância dos valores de rigidez $(\mathrm{N} / \mathrm{mm})$ das mandíbulas ..35

Tabela 5.2 - Médias de rigidez das mandíbulas $(\mathrm{N} / \mathrm{mm})$ da interação Angulação x Fase do teste ............................................................................ 35

Tabela 5.3 - Análise de variância dos valores de resistência à fratura $(\mathrm{N})$ das mandíbulas

Tabela 5.4 - Médias $(\mathrm{N})$ de resistência à fratura e valores críticos, pelo teste de Tukey para contrastes.

Anexo B - Valores dos resultados individuais e a estatística descritiva das amostras dos grupos I e II e das mandíbulas hígidas para rigidez e resistência a fratura (ver Anexo $B$, p.68) 


\section{LISTA DE ABREVIATURAS, SIGLAS E SÍMBOLOS}

$\begin{array}{ll}\mathrm{kg} & \text { Quilograma } \\ \mathrm{mm} & \text { Milímetro } \\ \mathrm{cm} & \text { Centímetro } \\ \mathrm{N} & \text { Newton } \\ \mathrm{FI} & \text { Fixação interna } \\ \mathrm{FIR} & \text { Fixação interna rígida } \\ \text { 3D } & \text { Três dimensões } \\ { }^{\circ} \mathrm{C} & \text { Grau Celsius } \\ \circ & \text { Grau } \\ \text { \$ } & \text { Dólar } \\ \text { PVC } & \text { Polivinilcloreto }\end{array}$




\section{SUMÁRIO}

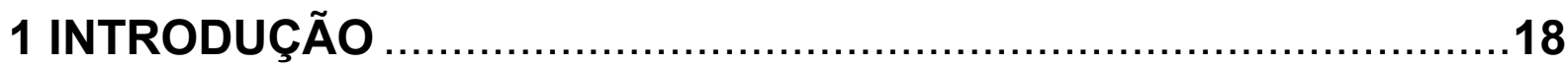

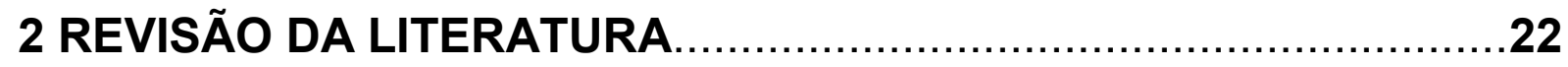

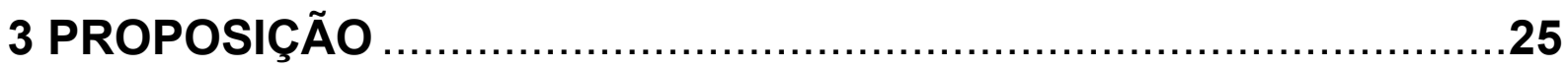

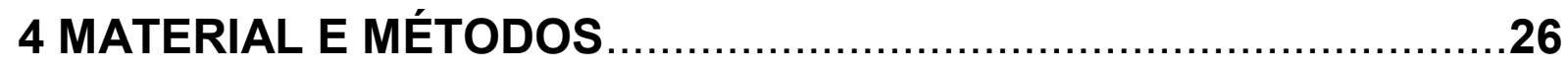

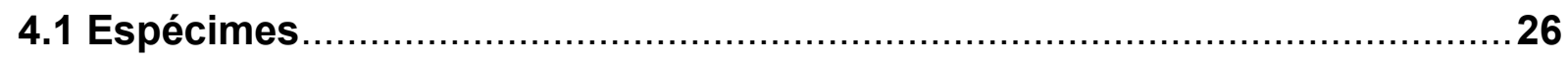

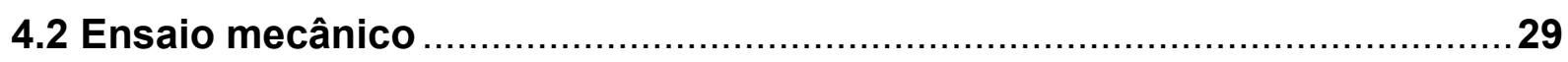

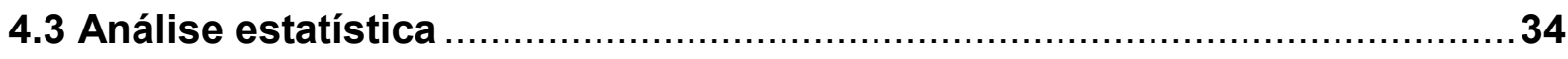

5 RESULTADOS

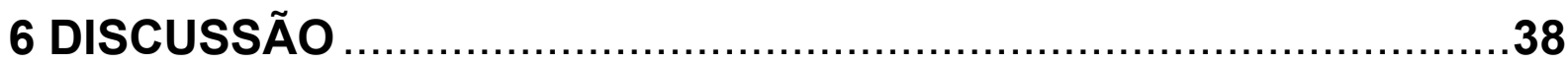

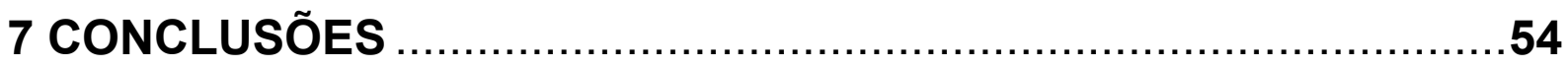

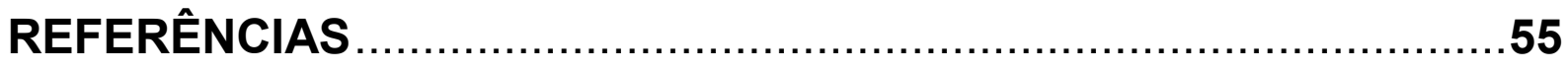

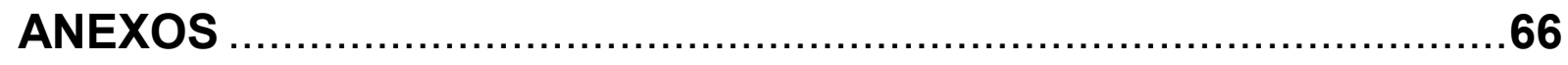




\section{INTRODUÇÃO}

Diferentes técnicas cirúrgicas têm sido usadas para o tratamento das deformidades dento esqueléticas, e atualmente, a osteotomia sagital bilateral do ramo mandibular é o procedimento mais utilizado pelos cirurgiões buco-maxilofaciais para correção de deformidades mandibulares (PROFFIT et al., 1989). Ela tem sido utilizada desde o final da década de cinqüenta, sendo inicialmente descrita por Trauner e Obwegeser (1957), e certamente representou grande avanço no tratamento das deformidades maxilo-faciais. A partir daí, várias modificações da técnica original foram propostas por diferentes autores, objetivando torná-la de execução mais fácil, diminuir sua morbidade e complicações, aumentar a sobreposição entre os segmentos com conseqüente melhora da estabilidade e minimizar o descolamento de tecidos moles (BELL; SCHENDEL, 1977; DAL PONT, 1961; EPKER, 1977; GALLO et al., 1976; HUNSUCK, 1968; WOLFORD; BENNETT; RAFFERTY, 1987).

Outro grande avanço na cirurgia maxilo-facial foi a introdução da fixação interna rígida, o que ofereceu uma série de vantagens em relação aos métodos tradicionais até então utilizados para fixação. Spiessl (1976) introduziu o uso de parafusos na fixação da osteotomia sagital. Em sua técnica original, o autor defendia a aplicação de parafusos compressivos (lag), utilizando três parafusos de 2,7 mm. Com o desenvolvimento das placas e parafusos de titânio, a fixação interna rígida (FIR) tem sido o método preferido na estabilização da osteotomia sagital bilateral do ramo mandibular, devido a ausência da necessidade do bloqueio maxilo-mandibular, maior comodidade ao paciente, rápido retorno a função pré-cirúrgica, melhor suporte 
nutricional, facilidade para higienização bucal, fácil manutenção das vias aéreas e redução das recidivas (HAUG; BARBER; PUNJABI, 1999; PETERSON; HAUG; VAN SICKELS, 2005).

Vários investigadores têm proposto métodos alternativos de fixação visando melhorias para este procedimento, tais como: diminuir distúrbios neurosensoriais como resultado de forças compressivas dos parafusos; diminuir deslocamento condilar médio-lateral e deslocamento súpero-inferior do segmento proximal; reduzir lesões ao feixe vásculo-nervoso alveolar inferior; dispensa de incisão percutânea para instalação dos parafusos (SCHWIMMER et al., 1994).

Avanços na técnica cirúrgica incluem o uso de fixação rígida com placas (CHAMPY et al., 1978; McDONALD, 1987; STOELINGA; BORSTLAP, 2003; TUCKER; OCHS, 1988) ou parafusos (DOUMA; KUFTINEC; MOSHIRI, 1991; ELLIS 3rd, 1992; ELLIS 3rd; REYNOLDS; CARLSON, 1988; FINN et al., 1980; FUJIOKA; FUJII; HIRANO, 2000; HUGHES; JORDAN, 1972; JETER; VAN SICKELS; DOLWICK, 1984; KREKMANOV; LILJA, 1986; LINDORF, 1986; MAURER; KNOLL; SCHUBERT, 2003; MOMMAERTS, 1991; NIEDERDELLMANN; SHETTY; COLLINS, 1987; PAULUS; STEINHAUSER, 1982; PERROTT et al., 1994; PUTNAM; BOUWMAN; TUINZING, 1993; SAKA, 2000; SCHWARTZ; RELLE, 1996; SHUFFORD; KRAUT, 1989; SOUYRIS, 1978; STEINHAUSER, 1982; TUCKER; OCHS, 1988; TUSLANE; SCHENDEL, 1989; VAN SICKELS, 1991; WATZKE et al., 1990) com o intuito de aumentar a estabilidade da fixação. A maioria das técnicas de fixação rígida com parafusos faz uso do acesso trans-bucal (GINGRASS; MESSER, 1986; KREKMANOV; LILJA, 1986; MULLER; BACH, 1989; STEINHAUSER, 1982). Uma pequena incisão percutânea para inserção trans-bucal da chave com o parafuso é feita na região do ramo ascendente e ângulo da mandíbula. Esta técnica 
facilita a colocação dos parafusos (STEINHAUSER, 1982), mas pode levar a possíveis seqüelas, como formação de cicatriz aparente, lesão ao nervo facial e trigêmeo, sangramento e dificuldades na remoção tardia dos parafusos (BOUWMAN et al., 1995; TELTZROW et al., 2005).

Uma maneira de se evitar estas complicações seria a utilização de técnica para inserção intra-oral dos parafusos, como recomendado por Turvey (1986) e Kempf (1987). A grande limitação relacionada com esta técnica seria a dificuldade na inserção e restrição da disposição dos parafusos. Com a técnica trans-oral, os parafusos são inseridos com angulação próxima de $90^{\circ}$ ou perpendicular ao longo eixo da superfície óssea da mandíbula. Já na técnica intra-oral os parafusos são inseridos com ângulo próximo de $60^{\circ}$ em relação à superfície lateral da mandíbula.

Nas últimas décadas, vários estudos clínicos e laboratoriais têm sido realizados com a fixação da osteotomia sagital do ramo mandibular, analisando o tamanho dos parafusos (KOHN, 1996; SCHWIMMER et al., 1994), o uso de parafusos compressivos ou posicionais (BLOMQVIST et al., 1997; BLOMQVIST; ISAKSSON, 1994; MOMMAERTS, 1991; NIEDERDELLMANN; SHETTY; COLLINS, 1987; SHUFFORD; KRAUT, 1989; WATZKE; TUCKER; TURVEY, 1991), parafusos feitos de diferentes materiais (BOUWMAN; TUINZING, 1999; BOUWMAN; TUINZING; KOSTENSE, 1994; FERRETTI; REYNEKE, 2002; WITTENBERG; WITTENBERG; HIPP, 1991), e parafusos colocados em diferentes configurações (FOLEY; BECKMAN, 1992; HAMMER et al., 1995; KOHN, 1996; OBEID; LINDQUIST, 1991), mas poucos estudos avaliam a fixação utilizando diferentes angulações de inserção dos parafusos (FOLEY et al., 1989; KIM et al., 1993; UCKAN et al., 2001). 
Visto que, poucos trabalhos avaliaram a fixação da osteotomia sagital mandibular utilizando parafusos com diferentes angulações de inserção, o objetivo deste trabalho foi avaliar a resistência mecânica na fixação da osteotomia sagital em mandíbulas de carneiro, utilizando parafusos bicorticais posicionais inseridos com angulação próxima de $60^{\circ}$ ou $90^{\circ}$ simulando, respectivamente, as técnicas intra e extra-oral. 


\section{REVISÃO DA LITERATURA}

Revisando a literatura, encontramos inúmeros trabalhos laboratoriais e clínicos que avaliaram as técnicas de fixação interna rígida na osteotomia sagital mandibular. Destes, porém, poucos avaliaram a FIR com ênfase na técnica intraoral. Selecionamos e expomos alguns que apresentaram, ao nosso entendimento, grande importância na evolução das técnicas de fixação. Lembrando que o foco principal da revisão seria a fixação intra-oral, e esta sim, foi detalhadamente revisada e exposta mais adiante, embora que dentre a literatura pesquisada, poucos trabalhos versam sobre o assunto.

Ardary et al. (1989) realizaram estudo experimental em mandíbulas de cadáver para avaliar a estabilidade da fixação da osteotomia sagital quando fixada com duas configurações dos parafusos ( $L$ invertido e linear). Verificaram que as mandíbulas fixadas com parafusos dispostos em forma tripoidal ( $L$ invertido) apresentaram maior rigidez quando comparadas com as mandíbulas fixadas com parafusos em configuração horizontal (linear). Esta diferença foi estatisticamente significante $(p<0.01)$.

Schwimmer et al. (1994) fizeram estudo experimental comparando a resistência da fixação utilizando parafusos de $2,0 \mathrm{~mm}$ ou $2,7 \mathrm{~mm}$, posicionais e compressivos. Não obtiveram diferença estatisticamente significante entre os diferentes grupos. A variável primária que determinou a estabilidade foi a qualidade do osso subjacente.

Foley et al. (1989) avaliaram várias formas de fixação utilizando parafusos posicionais, compressivos e fio de aço. Verificaram que as osteotomias fixadas com 
parafusos colocados em forma de "L invertido" foram significantemente mais rígidas que a fixação com parafusos em forma linear ou fio $\mathrm{K}$ em forma triangular $(p<0.05)$. Não houve diferença significante entre parafusos compressivos e posicionais colocados na mesma configuração. No mesmo experimento, os autores não verificaram diferença significante na força transversa entre parafusos colocados em ângulo de $60^{\circ}$ ou $90^{\circ}$, com mesma configuração. Defendem que a decisão de utilizar uma técnica ou outra não deve ser baseada na resistência mecânica, e sim na experiência do cirurgião e uso da técnica mais segura.

Turvey (1986) defende a colocação intra-oral de três parafusos bicorticais. Os parafusos são inseridos obliquamente na região retromolar ao longo do ramo mandibular. Com esta técnica não há necessidade de incisão em pele, mas pelo acesso limitado não oferece a diversidade de opções para colocação dos parafusos, como na técnica trans-oral. O autor defende que a necessidade de inserção oblíqua dos parafusos quando na técnica intra-oral pode oferecer um fator adicional de força pelo fato dos parafusos ficarem em maior contato com osso cortical.

Kempf (1987) descreveu técnica similar de inserção intra-oral. O autor cita como vantagens da técnica um menor tempo operatório, menor quantidade de instrumentos e elimina a necessidade de incisão em pele.

Shepherd, Dohvoma e Harradine (1991) descreveram técnica de fixação da osteotomia sagital via acesso intra-oral, utilizando dois parafusos não compressivos. Em sua série de 10 pacientes operados, observaram índices de recidiva semelhantes aos encontrados utilizando a técnica trans-bucal. Concluíram que a técnica sugere ser estável, e que a osteotomia sagital pode ser fixada sem a necessidade de incisões em pele. 
Farole (1992) relatou técnica para inserção dos parafusos na fixação da osteotomia sagital mandibular via acesso intra-oral utilizando contra ângulo em peça de mão para realizar a perfuração e inserção dos parafusos. Defende como importantes vantagens da técnica a inserção intra-oral e perpendicular dos parafusos, rapidez e acuracidade na colocação dos parafusos, inclusive com torque padrão.

Kim et al. (1993) realizaram análise fotoelástica para verificar a distribuição de forças na fixação da osteotomia sagital em modelo experimental. Utilizaram diferentes configurações para inserção dos parafusos, sendo triangular e linear em $90^{\circ}$ ou oblíqua. Verificaram que a inserção oblíqua linear apresentou a menor distribuição do stress, comparada aos outros grupos.

O mesmo grupo de autores (KIM; ESSAKI; KAMEYAMA, 1995) realizaram estudo em mandíbulas de cadáver, com objetivo de avaliar a rigidez de três diferentes padrões de inserção dos parafusos na fixação da osteotomia sagital mandibular. Verificaram que a inserção linear oblíqua dos parafusos obteve maior resistência que a inserção linear em $90^{\circ}$, e menor resistência que o padrão triangular em $90^{\circ}$. Como conclusões, referem que os resultados obtidos suportam a utilização da técnica intra-oral com inserção oblíqua, da mesma forma com a técnica com padrão triangular. Defendem ainda, que a técnica intra-oral deveria ser utilizada sempre que possível, justificando os melhores resultados estéticos.

Uckan et al. (2001) realizaram estudo em mandíbulas de carneiro para comparar a estabilidade mecânica na fixação da osteotomia sagital obtida com parafusos bicorticais posicionais, dispostos de forma linear, com ângulo de inserção dos parafusos de $60^{\circ}$ e $90^{\circ}$. Como resultados, não encontraram diferença estatisticamente significante entre os grupos. 


\section{PROPOSIÇÃO}

Avaliar comparativamente o comportamento biomecânico de mandíbulas hígidas, com o de mandíbulas osteotomizadas e fixadas com parafusos angulados em $60^{\circ}$ ou $90^{\circ}$ ( o que simula as técnicas intra e extra-oral de fixação da osteotomia sagital),em um novo sistema de suporte que imita as forças atuantes e a liberdade de movimentos de uma mandíbula em função mastigatória.

A hipótese é que as mandíbulas osteotomizadas e fixadas com parafusos em ângulo próximo de $60^{\circ}$ ( que simula a inserção intra-oral dos parafusos), apresentariam comportamento semelhante às fixadas mediante inserção extra-oral comumente preconizada, a $90^{\circ}$. Desta forma, poderiam ser utilizadas na prática cirúrgica, evitando as complicações do acesso extra-oral. 


\section{MATERIAL E MÉTODOS}

Antes do início do presente estudo, obteve-se aprovação do Comitê de Ética em Pesquisa (Anexo A).

\subsection{Espécimes}

Foram selecionadas 10 mandíbulas frescas de carneiros com peso e idade semelhantes, adquiridas em frigorífico. As mandíbulas foram dissecadas com completa remoção das estruturas moles (músculos, periósteo, etc.) e lavadas em água corrente. Os processos coronóides foram removidos bilateralmente, para facilitar o posicionamento da mandíbula durante o experimento. A seleção final foi feita pela mensuração com paquímetro e padronização das distâncias borda posterior - forame mentual e borda inferior - cabeça do côndilo, como descrito por Foley e Beckman (1992). Para armazenagem, foram embaladas em sacos plásticos (polietileno) e mantidas sob refrigeração $\left(-20^{\circ} \mathrm{C}\right)$ até cinco horas antes da realização do experimento, ficando armazenadas em solução fisiológica até a realização das osteotomias.

Depois de serem descongeladas, as mandíbulas foram numeradas e receberam fixação com dois parafusos de ferro comum com rosca soberba colocados em perfurações feitas com broca para baixa rotação número 702 , na região anterior ao forame mentual. Os parafusos foram adicionalmente estabilizados com resina acrílica adaptada sobre uma meia-cana de tubo PVC rígido de $40 \mathrm{~mm}$ de 
diâmetro e quatro cm de comprimento. Esta meia-cana de PVC foi confeccionada sobre o dispositivo de ensaio mecânico, e serviria como apoio deslizante durante o próprio ensaio (Figura 4.1). Esta fixação teve a finalidade de manter rígidas as duas hemi-mandíbulas, visto que estes animais apresentam articulação fibrosa na região mediana da sínfise.

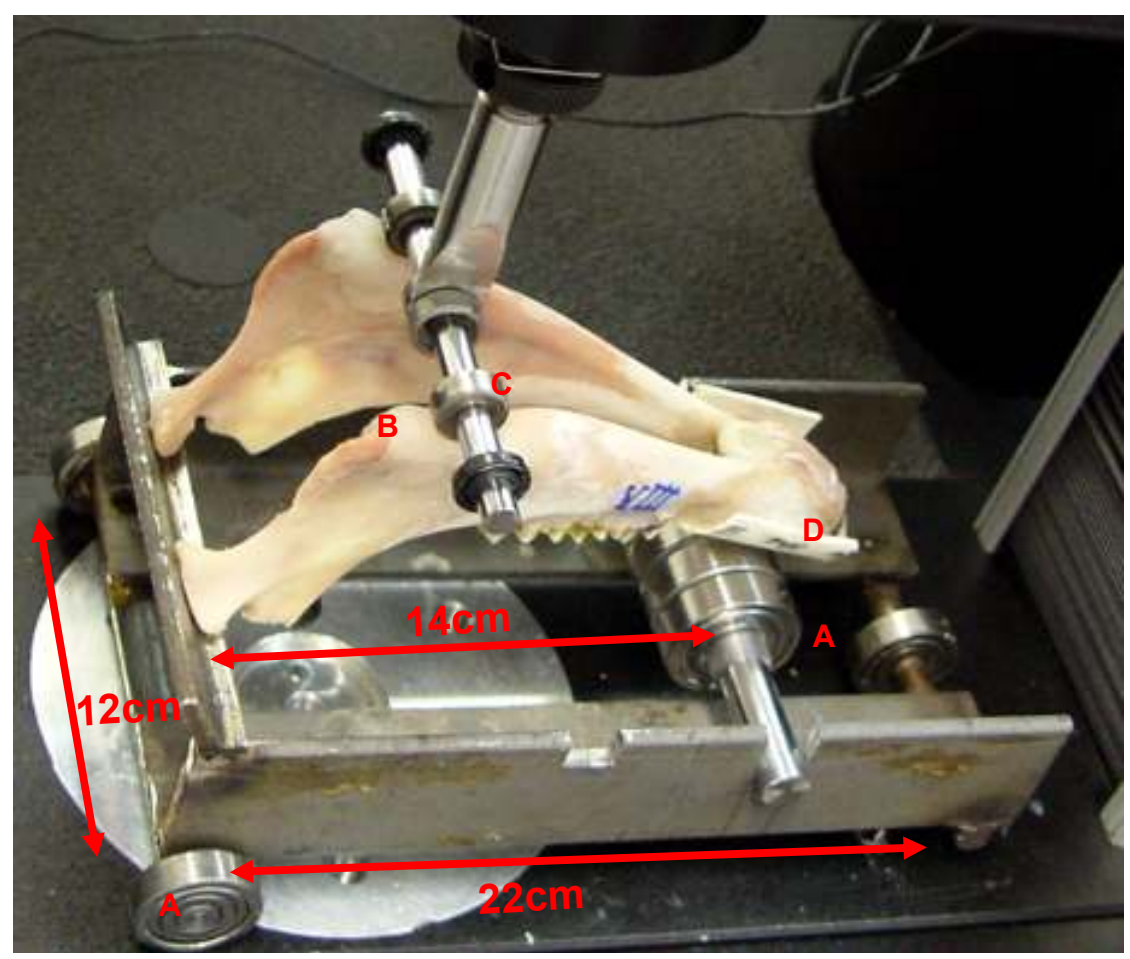

Figura 4.1 - Dispositivo metálico para teste. (A) Rolamentos. (B) Acréscimos de acrílico para descanso dos apoios deslizantes. (C) Apoios deslizantes. (D) Fixação da região mediana com parafusos e acréscimos de resina acrílica junto com tubo de PVC

A seguir, foi medida a rigidez de cada mandíbula, conforme explicado abaixo, na epígrafe "ensaio mecânico".

Imediatamente depois, as mandíbulas receberam osteotomia, fazendo uso de motor elétrico $\left(\right.$ Driller $^{\circledR}$ ) com serra reciprocicante na região de ramo e corpo mandibular, seguindo a técnica descrita por Trauner e Obwegeser (1957) com as modificações de Dal Pont (1961) e Hunsuck (1968). Após a osteotomia e remoção 
de interferências ósseas, o segmento distal foi avançado cinco milímetros bilateralmente e fixado com parafusos de titânio bicorticais posicionais do sistema 2,0 mm (MDT ${ }^{\circledR}$ - Rio Claro-SP), com disposição linear, em número de três para cada lado. Os terceiros molares foram removidos para não interferirem na fixação. Para as perfurações, foi utilizada broca longa com $1,6 \mathrm{~mm}$ de diâmetro conectada a uma peça reta em baixa rotação com irrigação contínua. Visando padronizar e minimizar possíveis variáveis, todas as osteotomias, perfurações e fixações foram feitas pelo mesmo pesquisador.

As mandíbulas foram distribuídas em dois grupos, constando de cinco espécimes cada:

- Grupo I (espécimes de I a V): as mandíbulas foram fixadas com parafusos inseridos em angulação de $60^{\circ}$ em relação à superfície óssea. Foram utilizados três parafusos para cada lado, dispostos de forma linear (Figura 4.2).

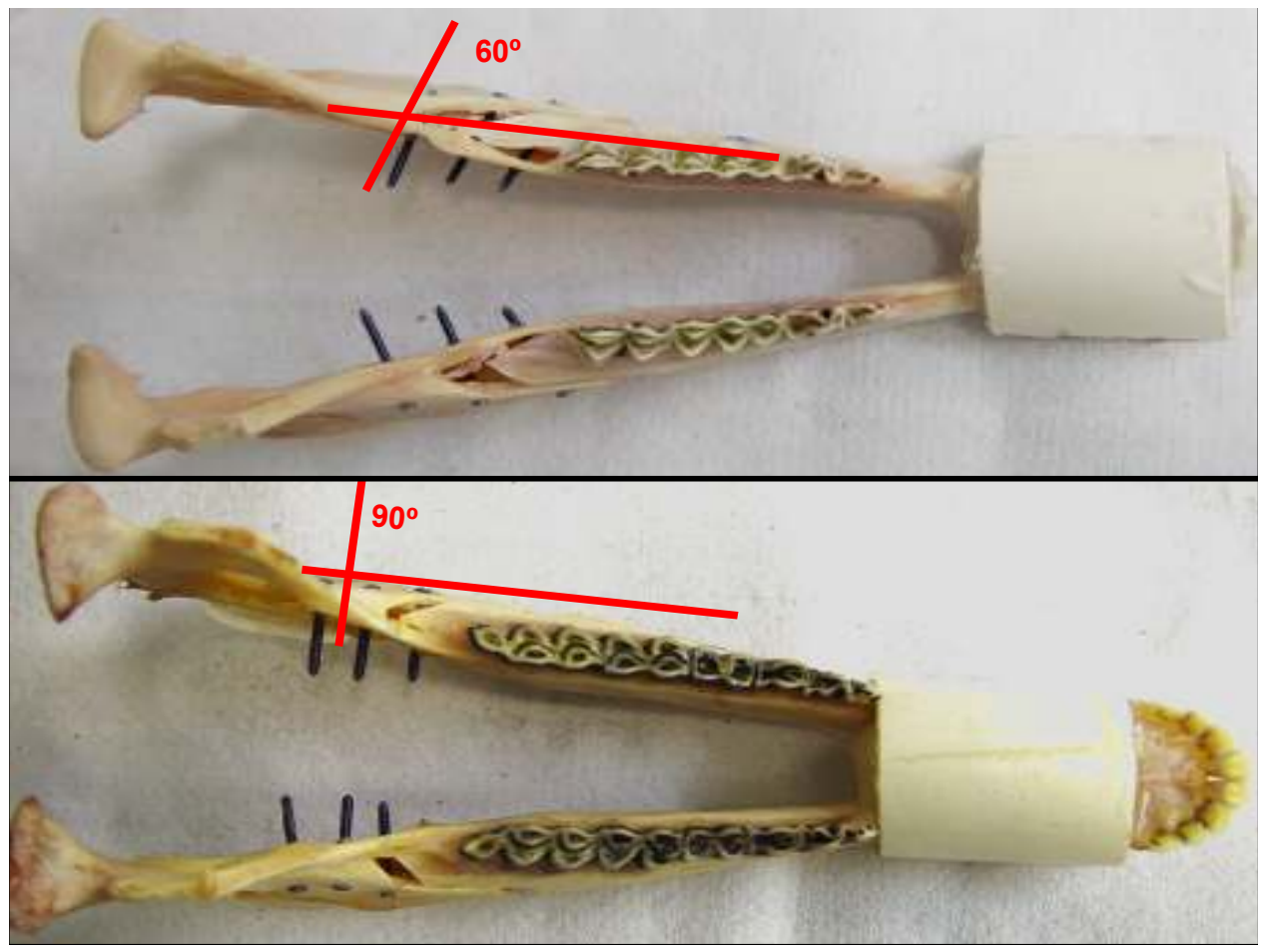

Figura 4.2 - Parafusos inseridos com ângulo próximos a $60^{\circ}$ ou $90^{\circ}$ em relação à superfície óssea, o que simularia respectivamente, as técnicas intra-oral e extra-oral 
- Grupo II (espécimes de VI a X): as mandíbulas foram fixadas com parafusos inseridos em angulação de $90^{\circ}$ em relação à superfície óssea. Foram utilizados três parafusos para cada lado, dispostos de forma linear (Figura 4.2).

\subsection{Ensaio mecânico}

Foi idealizado e construído um suporte metálico sobre rolamentos, o qual permitiu a movimentação e deformação das mandíbulas durante a aplicação da carga, de modo a imitar a liberdade de movimentos de uma mandíbula humana em função (encaixada na cavidade glenóide e livre em todas as outras regiões - Figuras 4.1 e 4.3). O posicionamento da mandíbula no dispositivo se deu com três pontos de apoio (côndilos e região anterior), e a aplicação da carga se deu, bilateralmente, na região entre o corpo e ângulo mandibular, área de maior inserção muscular, ou seja, região do músculo masseter, na direção das fibras do masseter (Figura 4.4). O dispositivo de aplicação de carga também dava liberdade para a movimentação independente dos dois pontos onde a carga era aplicada, tal como ocorreria numa mandíbula em função. 


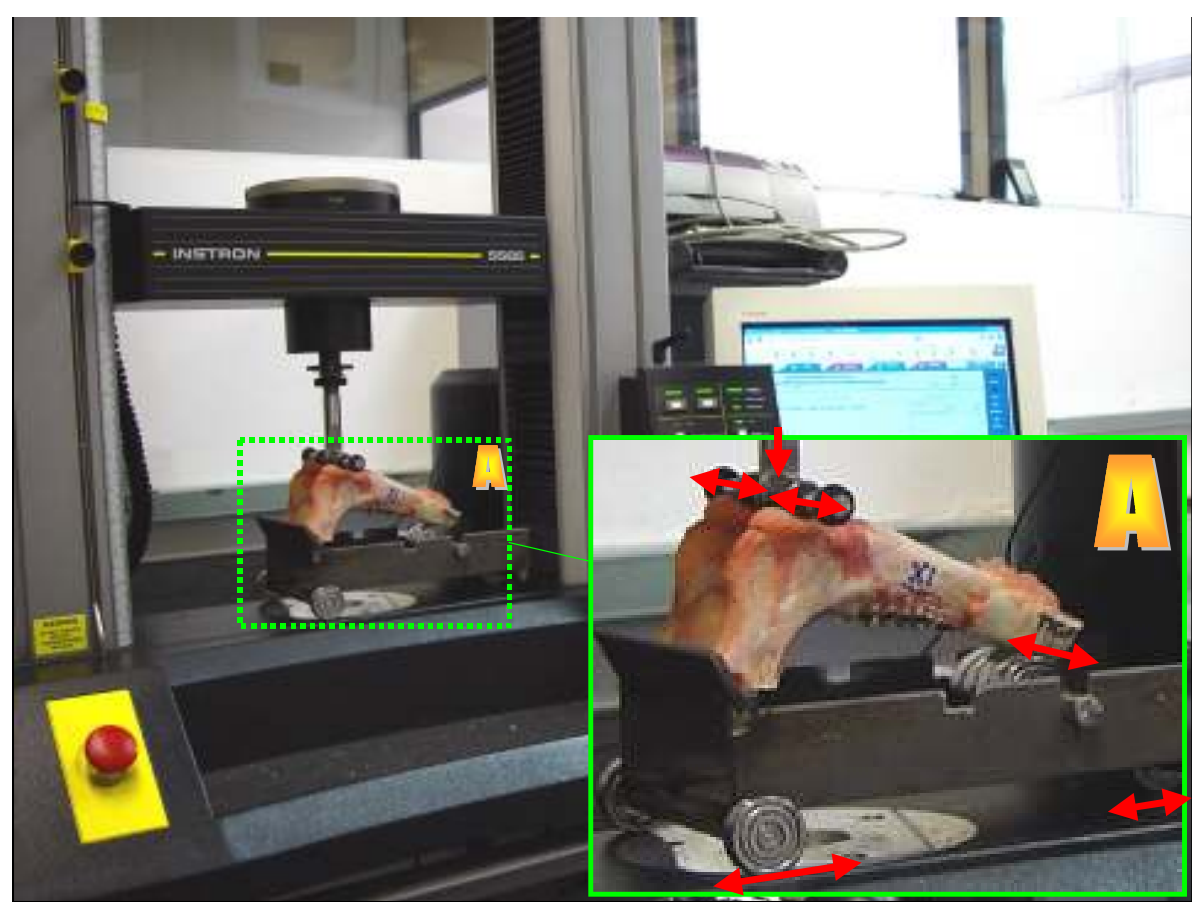

Figura 4.3 - Máquina Instron para realização do ensaio biomecânico. No detalhe, a mandíbula posicionada para a medição da rigidez. As setas de duas pontas indicam apoios que permitem liberdade de movimentos por giro ou deslizamento. A seta de uma ponta indica o ponto de aplicação da carga

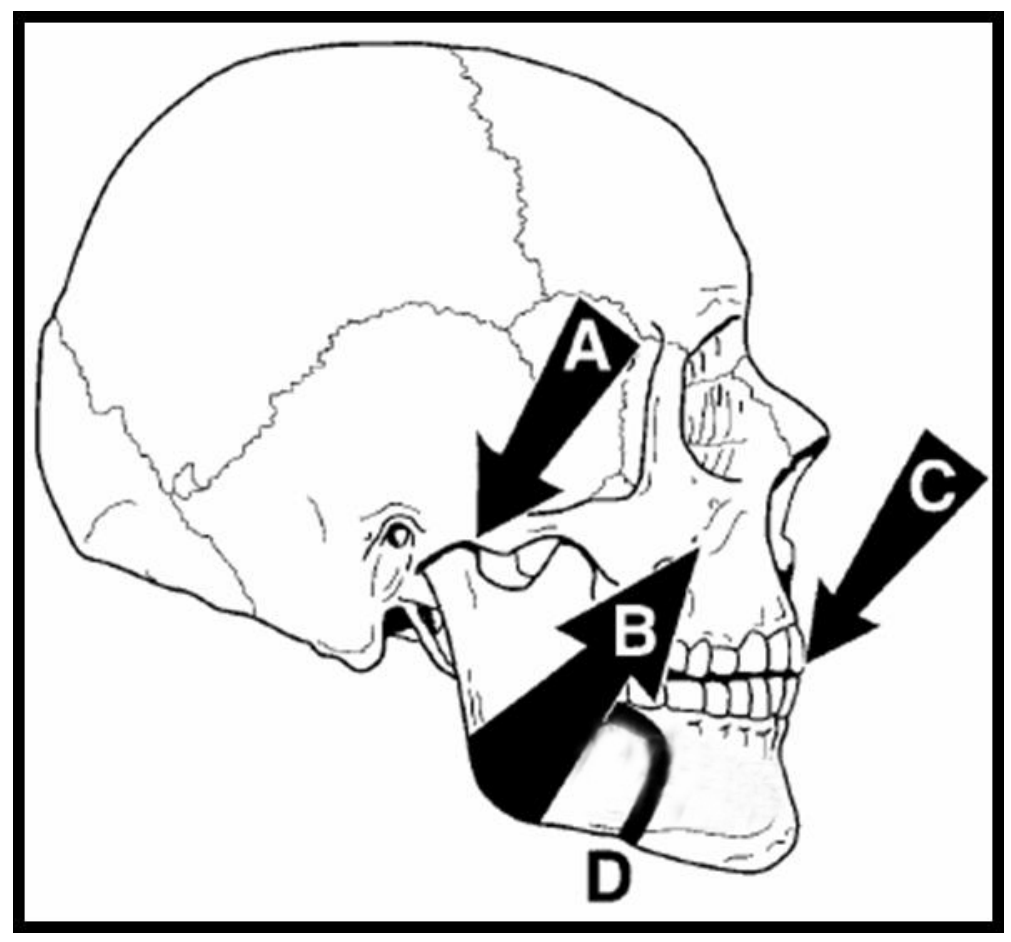

Figura 4.4 - Representação esquemática da mandíbula em função: existe um apoio no côndilo (A); a carga é aplicada no ângulo da mandíbula pela ação dos músculos mastigatórios maiores atuando sobre processo coronóide e ângulo mandibular (B); a resistência é exercida na região de implantação dos dentes $(C)$. A região do corpo mandibular onde foi realizada a osteotomia (D) e a fixação são submetidas a dobramento. (Adaptado de ARMSTRONG et al., 2001) 
Após o correto posicionamento da mandíbula e centralização do carro na base da máquina de ensaio universal (Instron 5565), iniciou-se o ensaio mecânico, o qual foi dividido em três etapas, que constou de:

\section{$1^{\text {a }}$ - Rigidez pré-osteotomia}

Antes de receberem a osteotomia sagital bilateral, todas as mandíbulas foram ensaiadas para obter sua rigidez. Para este ensaio foram aplicados três ciclos de deslocamento, numa velocidade de $0,07 \mathrm{~mm} / \mathrm{s}$ para o carregamento e $0,14 \mathrm{~mm} / \mathrm{s}$ para o descarregamento, com intervalo de 120 s entre cada ciclo. O primeiro deslocamento foi de $0,25 \mathrm{~mm}$, o segundo $0,5 \mathrm{~mm}$ e o terceiro $0,75 \mathrm{~mm}$ (Figura 4.5).

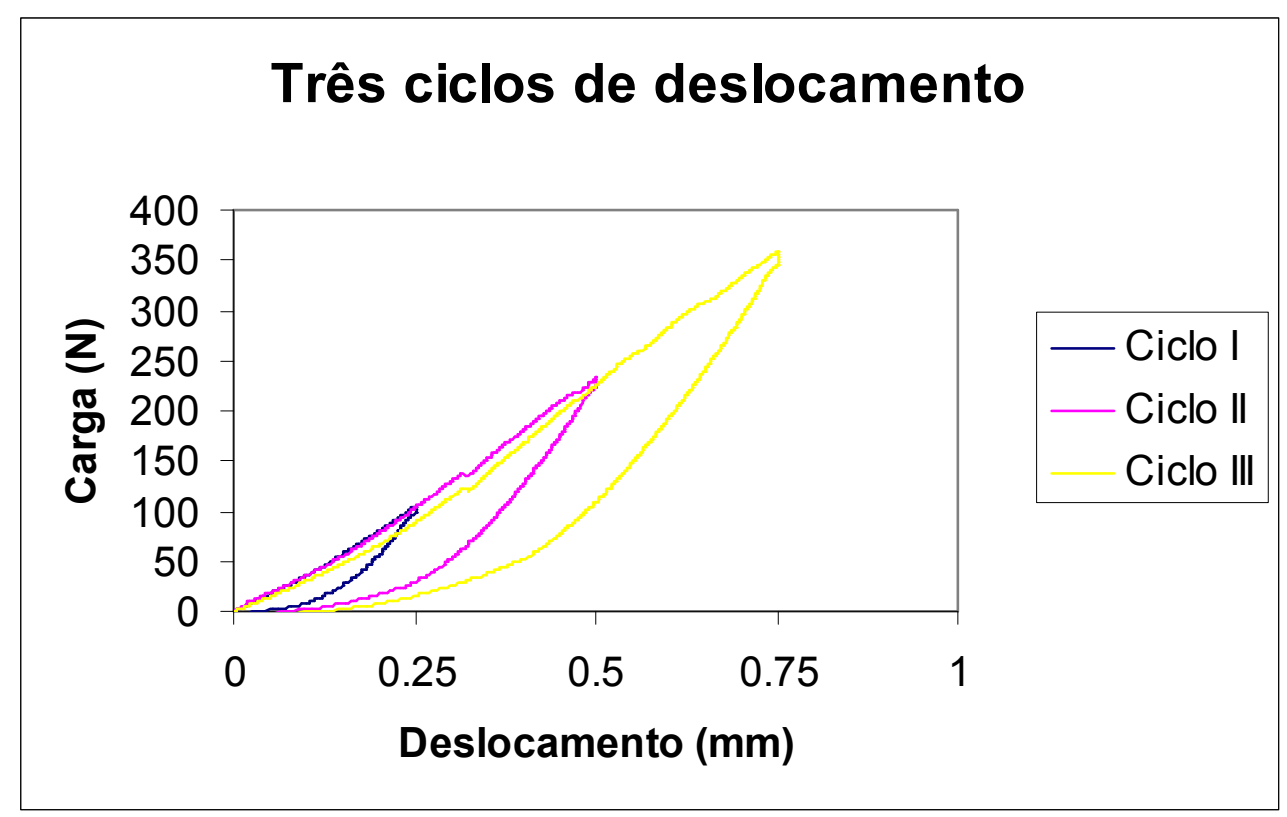

Figura 4.5 - Gráfico demonstrando os três ciclos de carregamento e descarregamento 
Os ciclos foram aplicados para garantir o perfeito assentamento da mandíbula no dispositivo de ensaio, e o tempo de espera entre ciclos foi adotado ao verificar-se que a total recuperação da deformação elástica da mandíbula não ocorria imediatamente depois de removida a carga (o que caracteriza um material viscoelástico). O tempo de espera para que a mandíbula retornasse à posição inicial foi inferior a dois minutos, então adotamos este valor como padrão. Para cálculo da rigidez da mandíbula foi adotado como padrão o terceiro ciclo, visto que este apresentou melhor uniformidade no traçado do gráfico, possivelmente devido a uma melhor acomodação da mandíbula sobre o suporte metálico.

Como valor de rigidez foi adotado o coeficiente angular (o número que multiplica o valor de $\mathrm{x}$ ) da reta ajustada aos pontos correspondentes ao carregamento na curva carga versus deslocamento. Adotamos a fórmula $y=a x$, onde $y=$ carga; $a=$ rigidez; $x=$ deslocamento (Figura 5.6). Ou seja, quanto maior a carga necessária para causar um determinado deslocamento, maior será a rigidez.

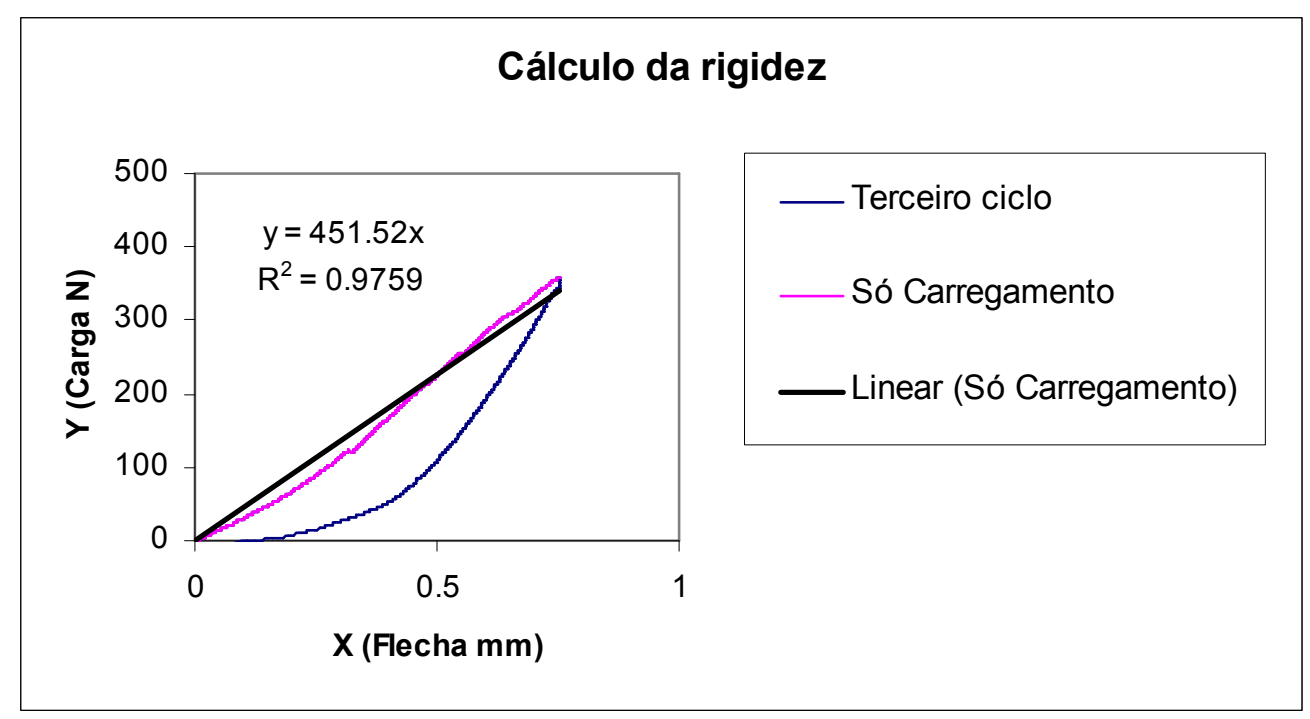

Figura 4.6 - Método de cálculo da rigidez: coeficiente angular da reta ajustada à região do carregamento do terceiro ciclo; neste caso $451,52 \mathrm{~N} / \mathrm{mm}$ 


\section{$2^{a}$ - Rigidez pós-osteotomia}

Após serem osteotomizadas e fixadas com avanço bilateral de cinco milímetros, as mandíbulas passaram pelo mesmo ensaio da epígrafe anterior e calculou-se um novo valor de rigidez.

\section{$3^{\mathrm{a}}$ - Resistência à ruptura ou à falha na fixação}

Imediatamente depois de se obter o valor de rigidez pós-osteotomia, as mandíbulas foram submetidas a carga compressiva com deslocamento contínuo de um milímetro por minuto, até que ocorresse rompimento ou falha na fixação. Foi adotado como critério para falha a diminuição abrupta de vinte por cento da carga aplicada sobre a mandíbula.

Duas mandíbulas hígidas também foram ensaiadas seguindo os mesmos parâmetros de ensaio, para se obter uma referência com a qual possam ser comparados os valores de resistência à ruptura das mandíbulas osteotomizadas. 


\subsection{Análise estatística}

Os valores de rigidez foram submetidos a análise de variância de medidas repetidas no mesmo espécime ("split-plot") tendo como fontes de variação a angulação dos parafusos (60 ou 90 graus) e a fase do teste (antes ou depois da osteotomia). As médias da interação foram contrastadas pelo teste de Tukey. Os valores de resistência à ruptura foram submetidos a análise de variância de uma única fonte de variação com três níveis desbalanceados (mandíbulas hígidas, reparadas com parafusos a 90 graus e reparadas com parafusos a 60 graus) e as médias contrastadas pelo teste de Tukey. 


\section{RESULTADOS}

A Tabela no Anexo B contém todos os resultados individuais obtidos das mandíbulas juntamente com a estatística descritiva.

As tabelas 5.1 a 5.4 apresentam as análises de variância e as tabelas de médias com os indicadores para contraste.

Tabela 5.1 - Análise de variância dos valores de rigidez (N/mm) das mandíbulas

\begin{tabular}{ccccc}
\hline $\begin{array}{c}\text { Fonte de } \\
\text { variação }\end{array}$ & G.L. & $\begin{array}{c}\text { Soma de } \\
\text { quadrados }\end{array}$ & $\begin{array}{c}\text { Quadrados } \\
\text { médios }\end{array}$ & Prob. (H0) \\
\hline $\begin{array}{c}\text { Angulação } \\
\text { do parafuso } \\
\text { Resíduo I }\end{array}$ & 1 & 127500 & 127500 & $95,301 \%$ \\
$\begin{array}{c}\text { Fase do } \\
\text { teste }\end{array}$ & 1 & 7342111250 & 7342111250 & $0,002 \%$ \\
$\begin{array}{c}\text { Interação } \\
\text { angulação x } \\
\text { fase }\end{array}$ & 1 & 25088750 & 25088750 & $56,265 \%$ \\
$\begin{array}{c}\text { Resíduo II } \\
\begin{array}{c}\text { Variação } \\
\text { total }\end{array}\end{array}$ & 8 & 294200000 & 36775000 & \\
\hline
\end{tabular}

Tabela 5.2 - Médias de rigidez das mandíbulas $(\mathrm{N} / \mathrm{mm})$ da interação Angulação x Fase do teste Letras iguais indicam que as diferenças não são significantes, pelo teste de Tukey (5\%) (valor de Tukey para contraste - 122,8)

\begin{tabular}{ccc}
\hline Angulação do parafuso $\left(^{\circ}\right)$ & \multicolumn{2}{c}{ Fase do experimento } \\
Inicial & Após osteotomia e fixação \\
\hline 60 & $516^{\mathrm{a}}$ & $111^{\mathrm{b}}$ \\
90 & $496^{\mathrm{a}}$ & $135^{\mathrm{b}}$ \\
\hline
\end{tabular}


Tabela 5.3 - Análise de variância dos valores de resistência à fratura $(\mathrm{N})$ das mandíbulas

\begin{tabular}{ccccc}
\hline $\begin{array}{c}\text { Fonte de } \\
\text { variação }\end{array}$ & G.L. & $\begin{array}{c}\text { Soma dos } \\
\text { quadrados }\end{array}$ & $\begin{array}{c}\text { Quadrados } \\
\text { médios }\end{array}$ & RQM \\
\hline Grupo & 2 & 6295392,2500 & 3147696,1250 & $284,54^{* * *}$ \\
Resíduo & 9 & 99562,0000 & 11062,4444 & \\
Total & 11 & 6394954,2500 & & \\
\cline { 2 - 4 } & significante ao nível de $0,1 \%$ & & &
\end{tabular}

Tabela 5.4 - Médias $(\mathrm{N})$ de resistência à fratura e valores críticos, pelo teste de Tukey para contrastes*

\begin{tabular}{ccc}
\hline Grupo & $\mathbf{n}$ & Resistência à fratura $(\mathbf{N})^{* *}$ \\
\hline $60^{\circ}$ & 5 & $278^{\mathrm{C}}$ \\
$90^{\circ}$ & 5 & $448^{\mathrm{B}}$ \\
Hígidas & 2 & $2295^{\mathrm{A}}$ \\
\hline
\end{tabular}

* Tukey $(5 \%)$ para 2 médias $(n=5)=150,5$

Tukey $(5 \%)$ para duas médias $(n=5$ e $n=2)=199,1$

** Médias com letras diferentes são significantemente diferentes entre si $(p \leq 5 \%)$

A rigidez das mandíbulas caiu significativamente após a osteotomia, sem diferenças significantes entre os grupos a sessenta ou noventa graus, pois a interação não foi significante. A angulação do parafuso não provocou diferenças significantes na rigidez da mandíbula, mas conduziu a uma resistência à fratura significativamente maior para os parafusos a 90 graus. Nenhuma das fixações com parafusos conseguiu restituir a resistência à fratura das mandíbulas hígidas.

A falha na fixação ocorreu por parafuso solto da cortical lingual (osso espanado) em sessenta por cento dos casos, seguido por fratura óssea e parafuso 
solto (Figura 5.1) em trinta por cento, e apenas fratura óssea, dez por cento. 0 padrão de falha se deu igual nos dois grupos estudados.

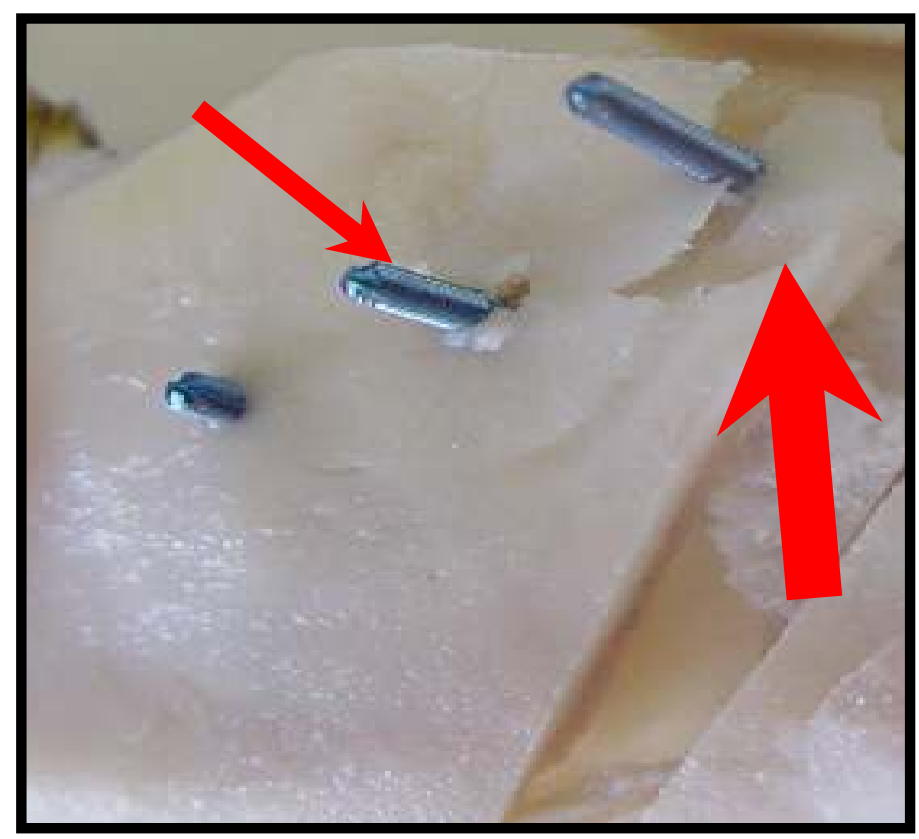

Figura 5.1 - Vista medial do ramo mandibular mostrando falha na fixação, evidenciando parafuso solto (seta estreita) e fratura óssea (seta larga) 


\section{DISCUSSÃO}

Antes mesmo que as interpretações dos resultados possam ser feitas, achamos oportuno que as limitações do modelo de estudo devam ser discutidas. Os estudos in vitro dos sistemas de fixação interna (FI) em osteotomia mandibular fazem uso de diferentes tipos de materiais. Revisando a literatura, podemos verificar que existem vários tipos de modelo disponíveis que podem ser utilizados em ensaios biomecânicos: mandibula humana cadavérica (ARDARY et al., 1989; KIM; ESSAKI; KAMEYAMA, 1995; KOHN et al., 1995; SCHWIMMER et al., 1994; THROCKMORTON et al., 1992), mandíbula de animais, como carneiro, macaco e cão (BOSANQUET; GOSS, 1987; FOLEY; BECKMAN, 1992; GOMES; GUIMARÃES FILHO; MAZZONETTO, 2003; MAYO; ELLIS 3rd, 1987; NISSENBAUM; LOWNIE; CLEATON-JONES, 1997; UCKAN et al., 2001), costela bovina, réplicas sintéticas de poliuretano ou mesmo madeira, dentre outros (BREDBENNER; HAUG, 2000; CHOI et al., 1995; ERKMEN et al., 2005a, 2005b; FOLEY, 1994; FOLEY et al., 1989; HAUG, 1996; KIM et al., 1993; KOHN et al., 1995; MAURER; HOLWEG; SCHUBERT, 1999; MURPHY; HAUG; BARBER, 1997; OZDEN et al., 2006; SHETTY, 1997; SHETTY et al., 1996a, 1996b; THARANON, 1998; VAN SICKELS et al., 2005). Todos estes apresentam suas vantagens e desvantagens, sendo que a escolha do método dependerá da experiência e particularidades do pesquisador. A mandíbula humana, embora seja aparentemente a mais indicada, tem como grande desvantagem a dificuldade na obtenção de vários espécimes, além de não haver padronização anatômica. Particularmente devido a algumas limitações na obtenção de um modelo ideal, acreditamos que num estudo onde há necessidade de vários 
espécimes, devemos utilizar modelo que seja barato, disponível e reproduzível para o experimento em questão.

As mandíbulas de animais podem ser conseguidas mais facilmente, com custo reduzido, dependendo do animal utilizado. Dentre elas, a que é mais frequentemente utilizada em ensaios mecânicos seria a mandíbula de carneiro. As réplicas sintéticas disponíveis nos oferecem adequado substituto, seguindo os padrões anatômicos e estruturais do osso humano. Elas apresentam cortical densa e compacta, preenchida por medular porosa, semelhante ao encontrado na mandíbula humana. Isto proporciona características semelhantes ao osso. Outra grande vantagem dos modelos sintéticos é a padronização anatômica, onde todos os espécimes seguem uma mesma forma estrutural. Como grande desvantagem, podemos citar o custo elevado destes modelos.

Diante disto, o modelo adotado no presente trabalho foi mandíbula de carneiro fresca e congelada. Como principais vantagens, podemos citar a disponibilidade, baixo custo e facilidade na obtenção. Além dos requisitos favoráveis já discutidos, outro fator levado em consideração foi o fato de sua anatomia ser, a grosso modo, semelhante à anatomia humana. Ela tem sido utilizada e validada por diferentes autores, com sucesso, no estudo biomecânico da osteossíntese mandibular (FOLEY; BECKMAN, 1992; GOMES; GUIMARÃES FILHO; MAZZONETTO, 2003; NISSENBAUM; LOWNIE; CLEATON-JONES, 1997; UCKAN et al., 2001).

Um fator que pode ser questionado diz respeito ao tipo de osso utilizado, se desvitalizado fresco ou congelado. O osso desvitalizado (fresco ou congelado) poderia perder suas propriedades biomecânicas, desta forma interferir nos 
resultados do estudo. Outra dúvida seria em relação às propriedades mecânicas e elásticas do osso fresco versus congelado.

Evans (1973) relatou não haver diferenças significantes entre as propriedades mecânicas do osso fresco e osso congelado armazenado $\mathrm{a}-20^{\circ} \mathrm{C}$ retornando à temperatura corporal antes do teste. Notou também poucas diferenças mecânicas entre osso vivo e osso desvitalizado.

Ashman e Van Buskirk (1987) verificaram que o método de congelamento para armazenamento de autópsias de mandíbulas humanas se mostrou eficaz e adequado para manter as propriedades elásticas do tecido ósseo.

Baseado nestes achados e concordando com outros autores (FOLEY; BECKMAN, 1992; GOMES; GUIMARÃES FILHO; MAZZONETTO, 2003; NISSENBAUM; LOWNIE; CLEATON-JONES, 1997; UCKAN et al., 2001), acreditamos que este modelo seja apropriado, particularmente porque todos os espécimes foram armazenados pelo mesmo período de tempo antes do teste mecânico. As mandíbulas foram colocadas em sacos plásticos, onde se tomou cuidado para que elas permanecessem em estado úmido e hidratadas, sendo então congeladas e armazenadas $\mathrm{a}-20^{\circ} \mathrm{C}$.

Outro fator que deve ser levado em consideração, é que tipo de ensaio mecânico será realizado. Vários estudos têm utilizado modelos com apenas dois pontos de carga (cantilever), ou seja, um ponto em uma extremidade, onde o espécime é mantido fixado, e outro na extremidade oposta onde é aplicada a carga compressiva (ANUCUL; WAITE; LEMONS, 1992; CROFTS et al., 1990; FOLEY; BECKMAN, 1992; GOMES; GUIMARÃES FILHO; MAZZONETTO, 2003; HAUG, 1993; HAUG; BARBER; PUNJABI, 1999; HEGTVEDT; MICHAELS; BEALS, 1994; IKEMURA et al., 1984, 1988; LEE; PIECUCH, 1992; LOUKOTA; SHELTON, 1995; 
MURPHY; HAUG; BARBER, 1997). Entretanto, a biomecânica da mandíbula humana em função mastigatória é bem mais complexa que movimentos apenas verticais (THROCKMORTON; THROCKMORTON, 1985). Um modelo mais acurado para representar as forças sagitais aplicados na mandíbula em função é baseado na suposição da simetria, nos quais são criados modelos matemáticos para desenvolver estudo em elemento finito. Estes modelos conseguem representar as forças mastigatórias em três Dimensões (3D), mas são modelos teóricos, que não podem ser reproduzidos em ensaios biomecânicos in vitro (KOOLSTRA et al., 1988; THROCKMORTON; THROCKMORTON, 1985).

De acordo com Armstrong et al. (2001), o modelo de três pontos utilizado em seu estudo foi adequado e permitiu consistência na formação da curva carga versus deslocamento, além de gerar medidas reproduzíveis dos limites elásticos que puderam ser comparadas entre os diferentes tipos de fixação.

Concordando com Armstrong et al. (2001), acreditamos que um modelo mais adequado deva apresentar três pontos de carregamento, desta forma incorporando as três maiores forças atuantes sobre a mandíbula em função. $O$ esquema representado na Figura 4.4 evidencia as áreas de maior carga e suas direções. Baseado nesse esquema, o modelo adotado apresenta três pontos de carregamento, ou seja, um na região do côndilo, outro na região anterior, e por último, onde a carga é aplicada na região de ângulo mandibular (Figura 4.1). Neste estudo, utilizando o suporte sobre rodas, aproximou-se da mecânica mandibular em função, pois, diferente dos modelos anteriores, este suporte permitiu a dissipação das forças tridimensionais que foram geradas pela máquina de ensaio, deformação esta, que era absorvida pela haste de fixação utilizada nos modelos anteriores. 
Lembramos que o objetivo principal do estudo foi avaliar e comparar dois tipos de $\mathrm{FI}$ na osteotomia sagital simulada. Ele não reproduz o complexo comportamento da mandíbula humana quando em função e sua interação entre osso, músculo e outros tecidos.

Dentre todos os estudos que avaliam a biomecânica dos sistemas de fixação, existem controvérsias com relação ao critério adotado para avaliar a falha das fixações, pois a falha clínica pode ocorrer ainda antes da que ocorre num ensaio mecânico, o qual tem um ponto experimentalmente pré-definido como falha (ARMSTRONG et al., 2001). Entretanto, num modelo experimental essa falência do sistema de fixação pode ser definida como um ponto de inflexão entre a fase elástica e plástica na curva de carga versus deslocamento (EDWARDS; DAVID, 1996; HEGTVEDT; MICHAELS; BEALS, 1994; IKEMURA et al., 1984, 1988; THROCKMORTON; THROCKMORTON, 1985).

Sabe-se que há diminuição na força de mordida no período pós-operatório imediato em indivíduos submetidos a cirurgia ortognática ou tratamento de fraturas maxilo-mandibulares (ELLIS 3rd; THROCKMORTON; SINN, 1996). Isto é importante, como veremos mais adiante, devido ao fato de que mesmo um sistema de fixação sendo menos rígido e menos estável biomecanicamente, ele pode apresentar sucesso clínico tão bom quanto um sistema teoricamente mais resistente. Revisando tal assunto na literatura, encontramos alguns trabalhos que julgamos ser interessantes.

Gibbs et al. (2002) verificaram que a força máxima sobre as regiões do segundo pré-molar, primeiro e segundo molares teve média de 720 N com variação de 244 a $1243 \mathrm{~N}$ para pacientes totalmente dentados. 
Hagberg (1986) estimou em 364 Newtons o pico das forças mastigatórias em pacientes mastigando amêndoas.

Vários estudos têm demonstrado que depois de uma fratura ou cirurgia mandibular, há diminuição na força de mordida. Gerlach e Schwarz (2002) verificaram que uma semana após os pacientes terem sido tratados de fratura do ângulo mandibular, estes apresentavam apenas $31 \%$ da carga vertical máxima, quando comparados com grupo controle.

Ellis 3rd, Dechow e Carlson (1988) em estudo com animais verificaram que as forças de mordida foram aumentando gradativamente, mas ainda permaneceram menores, quando comparadas com os níveis pré-operatórios doze semanas após a cirurgia.

Outro trabalho deste mesmo grupo sugere que a força de mordida na região do molar, para indivíduos não operados foi em média $52,3 \mathrm{Kg}(512,5 \mathrm{~N})$ para homens e $39 \mathrm{Kg}(382,2 \mathrm{~N})$ para mulheres. Sua estimativa para a força de mordida na região de molar no período pós-operatório imediato foi de 21,5 a 48,1 Kg (210,7 a $471,38 \mathrm{~N})$ para homens e de 13,3 a 19,2 $\mathrm{Kg}(130,3$ a $188,2 \mathrm{~N})$ para mulheres (THROCKMORTON; BUSCHANG; ELLIS 3rd, 1996).

Harada et al. (2000) realizaram trabalho similar ao descrito acima, onde os investigadores avaliaram as forças de mordida no pré e pós-operatório de pacientes submetidos a cirurgia ortognática. Verificaram que as forças oclusais dos pacientes antes da cirurgia foram de 293,2 a $162 \mathrm{~N}$ para homens e 208,5 a 98,8 $\mathrm{N}$ para mulheres. Em duas semanas de pós-operatório, as forças oclusais foram de 60,8 a 41,5 N para homens e 69,6 a 29,4 N para mulheres. Já em oito semanas após a cirurgia, variou de 184,7 a $89 \mathrm{~N}$ e 199,1 a $88,5 \mathrm{~N}$, respectivamente. 
Dentre vários estudos in vitro que examinaram a estabilidade, ponto de falha na carga máxima, deslocamento e rigidez, todos demonstraram que a fixação com mini-placa e parafuso monocortical foi menos rígida, mais suscetível à deformação, falha e deslocamento quando comparadas à fixação com três parafusos bicorticais posicionais. Sugeriram que o uso exclusivo da fixação com mini-placas não promove estabilidade suficiente necessária para função precoce (ANUCUL; WAITE; LEMONS, 1992; MURPHY; HAUG; BARBER, 1997; SHETTY et al., 1996a, 1996b).

Por outro lado, ao contrário do que os estudos in vitro demonstram, estudos clínicos evidenciam sucesso tanto na fixação com três parafusos bicorticais quanto na fixação com mini-placas.

Blomqvist et al. (1997) avaliaram 60 pacientes que foram submetidos a avanço mandibular, os quais receberam fixação com três parafusos bicorticais posicionais (via acesso percutâneo) ou mini-placa com parafusos monocorticais. Os pacientes foram seguidos por período de seis meses. Do ponto de vista da estabilidade, não houve diferença estatisticamente significante entre os dois tipos de fixação, tanto na direção sagital quanto vertical.

Quando falamos da fixação com três parafusos bicorticais, existem algumas variações da técnica, como por exemplo: diâmetro dos parafusos (2,0 ou 2,7 mm); parafusos inseridos de forma compressiva (lag) ou não compressiva (posicional); angulação de inserção dos parafusos $\left(60^{\circ}\right.$ ou $\left.90^{\circ}\right)$; bem como a disposição em que são inseridos ( $L$ invertido ou linear).

Sabe-se que o "padrão ouro" na FIR da osteotomia sagital mandibular é a utilização de três parafusos bicorticais dispostos em $\mathrm{L}$ invertido. Estudos biomecânicos comprovam a maior rigidez e estabilidade desta fixação quando 
comparada com a disposição linear ou mini-placas (FOLEY et al., 1989; FOLEY; BECKMAN, 1992; OCHS, 2003; VAN SICKELS; RICHARDSON, 1996).

Com relação ao tamanho dos parafusos, foi demonstrado que a utilização de parafusos com maior diâmetro não apresentou vantagens em relação à utilização de parafusos menores, quando foram comparados o sistema 2,7 mm com o 2,0 mm (SCHWIMMER et al., 1994).

No que diz respeito ao tipo de fixação, em relação à estabilidade, se utilizando parafusos compressivos (lag) ou não-compressivos (posicionais), os trabalhos demonstram estabilidade pós-operatória similar entre as duas técnicas (WATZKE; TUCKER; TURVEY, 1991). Porém, sabe-se que a técnica não compressiva apresenta algumas vantagens, não em estabilidade, mas sim em alguns aspectos cirúrgicos, como não desencadear torque aos côndilos, não comprimir o feixe vásculo-nervoso alveolar inferior, entre outras.

Outro fator interessante a se considerar, e que é objetivo principal do presente trabalho, seria sobre a angulação de inserção dos parafusos, se inseridos por via extra-oral $\left(90^{\circ}\right)$ ou intra-oral $\left(60^{\circ}\right)$ e qual o efeito sobre a estabilidade biomecânica na fixação da osteotomia sagital mandibular.

Segundo publicações ortopédicas, a inserção perpendicular dos parafusos com relação à superfície óssea seria o desejável na fixação de fraturas ou osteotomias (SCHLIKE; PANJABI; WHITE 3rd, 1979; PERREN, 1992).

Schlike, Panjabi e White 3rd (1979) estudaram a ótima orientação de parafusos transfixantes e demonstraram que a orientação preferida, e a qual suportaria melhor as forças de dobra foi a orientação perpendicular ao longo eixo do osso. Da mesma forma, a maior resistência a forças de torsão foi conseguida com a 
orientação dos parafusos perpendicularmente ao longo eixo da fratura ou osteotomia.

Baseado nos trabalhos ortopédicos e referindo que as osteotomias sagitais estão sujeitas a forças de torsão e dobra, Schwimmer et al. (1994) relatam que a orientação dos parafusos preferencialmente deveria ser perpendicular ao longo eixo da mandíbula. Referem ainda, que parafusos inseridos obliquamente ao longo eixo da superfície óssea e da osteotomia não sejam resistentes para suportar essas forças que atuam sobre a mandíbula, e como resultado, a estabilidade da fixação poderia ser comprometida.

Concordamos com o autor na questão de que a inserção perpendicular apresentaria maior estabilidade, e isto já foi demonstrado experimentalmente em alguns trabalhos (PERREN, 1992; SCHLIKE; PANJABI; WHITE 3rd, 1979; SCHWIMMER et al., 1994; UCKAN et al., 2001). Porém, discordamos de que a inserção oblíqua não suporte as cargas atuantes sobre a mandíbula em função, com conseqüente comprometimento da fixação. Não podemos nem devemos afirmar o sucesso clínico da inserção angulada dos parafusos nesse tipo de fixação, mas os resultados preliminares de nosso experimento, embora tenham demonstrado diferenças e menor resistência dos parafusos angulados em relação aos inseridos perpendicularmente ao osso, foram satisfatórios. Da mesma forma, dados laboratoriais e clínicos são em favor de que a fixação oblíqua seria suficiente para suportar tais cargas. Diferentes autores descrevem, relatam e defendem a sua utilização.

Kim, Essaki e Kameyama (1995) concluíram que os resultados obtidos com a fixação linear oblíqua suportam o uso da técnica intra-oral, e defendem ainda, que 
esta seja utilizada sempre que possível, justificando os melhores resultados estéticos.

Matematicamente, um parafuso inserido perpendicularmente ao longo eixo da osteotomia proporciona o maior grau de resistência ao movimento, proporcionando cerca de $20 \%$ mais resistência ao deslocamento dos segmentos. Por outro lado, a inserção em $60^{\circ}$ apresenta maior área de contato em osso cortical, e este fator pode balancear a desvantagem teórica da inserção angulada com relação a inserção perpendicular (UCKAN et al., 2001).

Uckan et al. (2001) realizaram estudo em mandíbulas de carneiro para comparar a estabilidade mecânica na fixação da osteotomia sagital obtida com parafusos bicorticais, dispostos em forma linear, com ângulo de inserção de $90^{\circ} \mathrm{e}$ $60^{\circ}$. Como resultado, não encontraram diferença estatisticamente significante na resistência ao movimento entre o ângulo de inserção dos parafusos.

Resultado semelhante foi obtido com o presente trabalho. Apesar de que em nosso estudo foi demonstrado que o grupo fixado com parafusos angulados em $60^{\circ}$ apresentou menor resistência mecânica (média: 278 N) com relação ao grupo fixado com parafusos angulados em $90^{\circ}$ (média: $478 \mathrm{~N}$ ), acreditamos que o resultado foi satisfatório. Não houve diferença estatisticamente significante da rigidez entre o grupo I e II. Contudo, mesmo que os parafusos angulados em $60^{\circ}$ apresentaram menor resistência, acreditamos que este não seja um fator determinante que irá interferir no resultado clínico, visto que já foi demonstrado que mesmo técnicas onde a resistência foi ainda inferior a esta, como as mini-placas, obteve-se sucesso clínico semelhante (BLOMQVIST et al., 1997). Isto talvez se explique pelo fato de que os estudos biomecânicos in vitro avaliem apenas os métodos de fixação, comparando uma técnica com outra, no que diz respeito à estabilidade e rigidez, dentre outras 
variáveis, não avaliando desta forma, a complexa relação da FIR atuando na mandíbula em função.

Quanto às mandíbulas hígidas que foram submetidas ao ensaio, estas suportaram a carga compressiva e tiveram rompimento com média bastante superior (2295 N) quando comparadas com as mandíbulas osteotomizadas e fixadas (278 e $448 \mathrm{~N}$ ). Esta grande diferença se daria pelo fato de que, ao contrário das mandíbulas dos grupos I e II que após serem osteotomizadas e reabilitadas com o sistema de $\mathrm{FI}$, não perderam solução de continuidade. Embora que o sistema de FI apresente rigidez e estabilidade, esta não é a mesma da mandíbula hígida, que apresenta suas corticais intactas. Ambas mandíbulas hígidas apresentaram fratura na mesma região anatômica, ou seja, região de côndilo mandibular. Região esta que é considerada área de fragilidade da mandíbula, estando sujeita a fraturas. A literatura demonstra que a região com as maiores incidências de fraturas na mandíbula está localizada justamente na região de côndilo mandibular (ELLIS 3rd; MOSS; EL-ATTAR, 1985; OLSON et al., 1982). Estes dados suportam e justificam as fraturas que ocorreram nas mandíbulas hígidas do presente trabalho.

Embora resultados de estudos in vitro, testes laboratoriais e outros parâmetros indiquem o uso de três parafusos bicorticais na fixação da osteotomia sagital mandibular, vários levantamentos e seguimento de casos demonstram o sucesso clínico do uso de placas e parafusos monocorticais. Desta forma, não necessariamente o método que se mostrou mais rígido, mais estável ou que suportou maior carga compressiva seria o de maior sucesso clínico, ou ainda o que deveria ser empregado na pratica clínica. Toda técnica apresenta suas vantagens e desvantagens, e alguns fatores podem ser determinantes para a escolha da que seja mais adequada a ser empregada. Segundo Ochs (2003), os fatores que 
influenciam na escolha da técnica de fixação são: anatomia óssea; presença de terceiros molares; fraturas desfavoráveis ou traços de fratura em galho verde; desenho da osteotomia; tipo de movimento cirúrgico; posicionamento do segmento proximal; posição do nervo alveolar inferior; volume dos tecidos moles; custo; preferência do cirurgião.

Segundo ainda o mesmo autor (OCHS, 2003), dentre os pacientes candidatos ou submetidos a osteotomia sagital do ramo mandibular, há uma considerável variação na morfologia óssea do ângulo e ramo mandibular. Além dessas variações, que podem determinar o tipo e desenho da osteotomia que o cirurgião irá realizar, outros fatores podem interferir no tipo de fixação que deverá ser empregada. Por exemplo, alguns profissionais realizam osteotomia na base do corpo mandibular logo após a osteotomia vertical anterior, visando facilitar a separação dos segmentos proximal e distal. Isto algumas vezes resulta numa fratura ou separação (split) em forma de "J", onde parte da cortical lingual fica aderida ao segmento proximal. Desta forma, não há uma cortical medial basal abaixo do nervo alveolar inferior para que seja inserido o parafuso bicortical nesta região, no caso de uma fixação com três parafusos dispostos em "L" invertido. Com isso, o cirurgião deverá realizar a fixação com três parafusos dispostos de forma linear, ou seja, na borda superior ou então lançar mão de outra técnica, como placas e parafusos monocorticais. Já em outros casos, como em pacientes com o ângulo mandibular aberto e ramo curto, pode não haver osso suficiente na borda superior posteriormente ao ultimo molar, o que impossibilitaria a inserção de três parafusos nessa área. Diante disto, todos estes fatores são importantes e devem ser levados em consideração no plano de tratamento e escolha da técnica de fixação. 
Com base no que foi descrito acima, podemos dizer que a escolha de uma técnica ou outra não deve ser feita apenas por intuição ou preferência do cirurgião, mas sim, deve-se avaliar criteriosamente todos os fatores possíveis que irão favorecer ou não cada uma das técnicas, e mesmo que bem selecionada, devemos saber e estar aptos a realizar mudanças que possam ocorrer trans-operatoriamente.

Uma pergunta que poderíamos levantar, no que diz respeito à anatomia óssea, seria qual área da mandíbula apresenta melhores condições (espessura e osso cortical) para realizar a osteossíntese. Estudos anatômicos têm demonstrado que a porção mais espessa da placa óssea cortical, tanto lingual quanto bucal, esta localizada na borda superior do ramo logo atrás ao último molar (CARTER et al., 1991; SMITH et al., 1991a, 1991b). Com isso, sabemos que esta área apresentando corticais mais espessas seria local ideal para inserção dos parafusos. Justamente nesta região são inseridos os parafusos quando da utilização da técnica intra-oral.

Um fator que podemos levar em consideração em favor do uso de parafusos na FIR da osteotomia sagital, seria com relação ao custo do material de fixação. Visto que a realidade em nosso país nem sempre é a desejável, e que em muitos serviços o fator financeiro pode pesar e ser até mesmo limitante, acreditamos que uma técnica que utilize menor quantidade de material com conseqüente redução de custos seja viável, não como variável principal, mas sim secundária. Em média, a utilização de placas e parafusos custaria cerca do dobro do valor se utilizarmos apenas parafusos bicorticais. O mesmo é exemplificado por Ochs (2003), onde o autor relata que em sua experiência, não há diferenças no tempo operatório quando da utilização de parafusos bicorticais ou mini-placas com parafusos monocorticais. A grande diferença estaria no custo do material, que em sua instituição, a lista de 
preços estaria entre $\$ 210$ (seis parafusos bicorticais) versus $\$ 430$ (duas miniplacas e oito parafusos monocorticais).

No caso de se optar por uma técnica intra-oral, fazendo uso de placa com parafusos monocorticais, um problema que poderia ocorrer seria a perda de um parafuso em um dos lados da osteotomia. Isso resultaria em rotação superior do segmento proximal, o que iria resultar em uma classe III com mordida aberta anterior no lado afetado. Para eliminar essa possibilidade, poderia ser utilizada placa com seis furos. Porém, necessitaria de placas com maior extensão, bem como maior espaço para ser adaptada, aumentando os custos do material de fixação (OCHS, 2003).

Segundo Ochs (2003), o uso de três parafusos bicorticais oferece o melhor custo benefício e rigidez previsível para a fixação da osteotomia sagital mandibular. Ainda segundo o autor, há numerosos padrões de inserção que podem ser utilizados, fazendo vantagem da localização, angulação e regiões de contato ósseo ou espaços onde estes possam ser inseridos. O padrão e seqüência de inserção dos parafusos devem ser individualizados trans-operatoriamente, para que haja uma otimização do resultado. Há situações selecionadas onde miniplacas, parafusos ou técnicas híbridas devem ser utilizadas. Como exemplo, em situações onde ocorreu fratura desfavorável ou grande defeito ósseo resultante da remoção simultânea do terceiro molar, deve-se optar pelo uso de miniplacas. O autor conclui que os cirurgiões devem estar aptos e bem versados em utilizar ambas as técnicas, além de terem habilidade para realizar ajustes intra-operatórios, dependendo do tipo de separação resultante da osteotomia e posição dos segmentos.

Como possíveis vantagens e desvantagens da técnica intra-oral, algumas que citamos e outras que são relatadas na literatura, estariam: 
- Vantagens: facilidade na remoção com anestesia local ou sedação, numa possível necessidade tardia causada por infecção ou outra causa; custo reduzido quando comparada com placas e parafusos. Em média, custaria em torno da metade do valor da fixação com placas e parafusos; simplicidade e menor tempo de execução; quantidade menor de instrumentais; ausência ou menores chances de lesão ao feixe vásculo nervoso alveolar inferior; maior engajamento e travamento entre parafusos e tecido ósseo cortical, devido à maior espessura cortical da região retromolar; ausência de riscos de lesionar o nervo facial; ausência de riscos de sangramento provenientes da incisão percutânea; ausência de incisão na pele. Esta seria, em nossa opinião, a principal vantagem da técnica, visto que não há riscos de formação de cicatriz aparente em área visível, principalmente em pacientes afrodescendentes, os quais apresentam tendência para desenvolver cicatriz hipertrófica ou quelóides.

- Desvantagens: dificuldade de inserção dos parafusos; risco de torque nos segmentos osteotomizados; acesso limitado, o que acarretaria numa diminuição das opções para posicionamento dos parafusos;

Analisando os resultados obtidos e dentro das limitações do presente trabalho, pode-se verificar que houve diferença estatisticamente significante entre os grupos, quando avaliamos a resistência à ruptura. Baseados em nossos resultados e visto que outros autores defendem e indicam o uso clínico da técnica intra-oral, acreditamos que esta seja uma alternativa viável, com algumas vantagens consideráveis, mas há necessidade da realização de outros estudos, principalmente in vivo, visando avaliar o sucesso clínico entre as duas técnicas. Isto possibilitaria o 
estudo continuado nessa linha de pesquisa, com intuito de melhoria nas técnicas cirúrgicas disponíveis. Concluímos que o suporte metálico desenvolvido para manter as mandíbulas se mostrou eficaz. Acreditamos que desta forma os movimentos mandibulares possam ser melhor reproduzidos durante o ensaio mecânico. 


\section{CONCLUSÃO}

Baseados nos resultados e metodologia empregada, dentro das limitações do trabalho, pode-se concluir que:

1. As mandíbulas fixadas com parafusos angulados em $90^{\circ}$ apresentaram maior resistência mecânica;

2. Não houve diferença estatisticamente significante da rigidez entre os grupos I e II;

3. O suporte metálico sobre rodas desenvolvido para o experimento se mostrou eficaz;

4. Há necessidade de outros estudos visando avaliar o sucesso clínico entre as duas técnicas; 


\section{REFERÊNCIAS ${ }^{1}$}

Anucul B, Waite PD, Lemons JE. In vitro strength analysis of sagittal split osteotomy fixation: noncompression monocortical plates versus bicortical position screws. J Oral Maxillofac Surg 1992;50(12):1295-9.

Ardary WC, Tracy DJ, Brownridge II GW, Urata MM. Comparative evaluation of screw configuration on the stability of the sagittal split osteotomy. Oral Surg Oral Med Oral Pathol 1989;68(2):125-9.

Armstrong JE, Lapointe HJ, Hogg NJ, Kwok AD. Preliminary investigation of the biomechanics of internal fixation of sagittal split osteotomies with miniplates using a newly designed in vitro testing model. J Oral Maxillofac Surg 2001;59(2):191-5.

Ashman RB, Van Buskirk WC. The elastic properties of a human mandible. Adv Dent Res 1987;1:64-7.

Bell WH, Schendel SA. Biologic basis for modification of the sagittal ramus split operation. J Oral Surg 1977;35(5):362-9.

Blomqvist JE, Ahlberg G, Isaksson S, Swartz K. A comparison of skeletal stability after mandibular advancement and use of two rigid internal fixation techniques. $J$ Oral Maxillofac Surg 1997;55:568-74.

Blomqvist JE, Isaksson S. Skeletal stability after mandibular advancement: a comparison of two rigid internal fixation techniques. J Oral Maxillofac Surg 1994;52(11):1133-7.

Bosanquet AG, Goss AN. The sheep as a model for temporomandibular joint surgery. Int J Oral Maxillofac Surg 1987;16(5):600-3.

Bouwman JP, Husak A, Putnam GD, Becking AG, Tuinzing DB. Screw fixation following bilateral sagittal ramus osteotomy for mandibular advancementcomplications in 700 consecutive cases. Br J Oral Maxillofac Surg 1995;33(4):231-4.

\footnotetext{
${ }^{1}$ De acordo com Estilo Vancouver. Abreviatura de periódicos segundo base de dados MEDLINE.
} 
Bouwman JP, Tuinzing DB. Biodegradeble osteosyntesis in mandibular advancement: a pilot study. Br J Oral Maxillofac Surg 1999;37:6-10.

Bouwman JP, Tuinzing DB, Kostense PJ. A comparative in vitro study on fixation of sagittal split osteotomies with Wurzburg screws, Champy miniplates, and Biofix (biodegradable) rods. Int J Oral Maxillofac Surg 1994;23:46-8.

Bredbenner TL, Haug RH. Substitutes for human cadaveric bone in maxillofacial rigid fixation research. Oral Surg Oral Med Oral Pathol Oral Radiol Endod 2000;90(5):57480.

Carter TB, Frost DE, Tucker MR, Zuniga JR. Cortical thickness in human mandibles: clinical relevance to the sagittal split ramus osteotomy. Int J Adult Orthod Orthognath Surg 1991;6(4):257-60.

Champy M, Lodde JP, Schmitt R, Jaeger JH, Muster D. Mandibular osteosynthesis by miniature screwed plates via a buccal approach. J Maxillofac Surg 1978;6:14-21.

Choi BH, Yoo JH, Kim KN, Kang HS. Stability testing of a two miniplate fixation technique for mandibular angle fractures. An in vitro study. J Craniomaxillofac Surg 1995;23(2):123-5.

Crofts CE, Trowbridge A, Maung Aung T, Brook IM. A comparative in vitro study of fixation of mandibular fractures with paraskeletal clamps or screw plates. J Oral Maxillofac Surg 1990;48(5):461-6.

Dal Pont G. Retromolar osteotomy for the correction of prognathism. J Oral Surg Anesth Hosp Dent Serv 1961;19:42-7.

Douma E, Kuftinec MM, Moshiri F. A comparative study of stability after mandibular advancement surgery. Am J Orthod Dentofacial Orthop 1991;100(2):141-55.

Edwards TJ, David DJ.A comparative study of miniplates used in the treatment of mandibular fractures. Plast Reconstr Surg 1996;97(6):1150-7.

Ellis E 3rd. Rigid fixation in orthognathic surgery. Selected Read Oral Maxillofac Surg 1992;2:1-17. 
Ellis E 3rd, Dechow PC, Carlson DS. A comparison of stimulated bite force after mandibular advancement using rigid and nonrigid fixation. J Oral Maxillofac Surg 1988;46:26-32.

Ellis E 3rd, Moss KF, el-Attar A. Ten years of mandibular fractures: an analysis of 2,137 cases. Oral Surg Oral Med Oral Pathol 1985;59(2):120-9.

Ellis E 3rd, Reynolds S, Carlson DS. Stability of the mandible following advancement: a comparison of three postsurgical fixation techniques. Am J Orthod Dentofacial Orthop 1988;94:38-49.

Ellis E 3rd, Throckmorton GS, Sinn DP. Bite forces before and after surgical correction of mandibular prognathism. J Oral Maxillofac Surg 1996;54(2):176-81.

Epker BN. Modifications in the sagittal osteotomy of the mandible. J Oral Surg 1977;35:157-9.

Erkmen E, Simsek B, Yucel E, Kurt A. Comparison of different fixation methods following sagittal split ramus osteotomies using three-dimensional finite elements analysis. Part 1: advancement surgery-posterior loading. Int J Oral Maxillofac Surg 2005a;34(5):551-8.

Erkmen E, Simsek B, Yucel E, Kurt A. Three-dimensional finite element analysis used to compare methods of fixation after sagittal split ramus osteotomy: setback surgery-posterior loading. Br J Oral Maxillofac Surg 2005b;43(2):97-104.

Evans FG. Factors affecting the mechanical properties of bone. Bull N Y Acad Med 1973;49(9):751-64.

Farole A. An intraoral technique for transoral rigid fixation of the sagittal split ramus osteotomy. J Oral Maxillofac Surg 1992;50:422-3.

Ferretti C, Reyneke JP. Mandibular, sagittal split osteotomies fixed with biodegradable or titanium screws: a prospective, comparative study of postoperative stability. Oral Surg Oral Med Oral Pathol Oral Radiol Endod 2002;93(5):534-7.

Finn RA, Throckmorton GS, Bell WH, Legan HL. Biomechanical considerations in the surgical correction of mandibular deficiency. J Oral Surg 1980;38(4):257-64. 
Foley WL. Appropriate testing of fixation for the mandibular sagittal split osteotomy. J Oral Maxillofac Surg 1994;52(7):780-1.

Foley WL, Beckman TB. In vitro comparison of screws versus plate fixation in the sagittal split osteotomy. Int J Adult Orthod Orthognath Surg 1992;7:147-51.

Foley WL, Frost DE, Paulin WB, Tucker MR. Internal screw fixation: comparison of placement pattern and rigidity. J Oral Maxillofac Surg 1989;47:720-3.

Fujioka M, Fujii T, Hirano A. Comparative study of mandibular stability after sagittal split osteotomies: biocortical versus monocortical osteosynthesis. Cleft Palate Craniofac J 2000;37(6):551-5.

Gallo WJ, Moss M, Gaul JV, Shapiro D. Modification of the sagittal ramus-split osteotomy for retrognathia. J Oral Surg 1976;34(2):178-9.

Gerlach KL, Schwarz A.Bite forces in patients after treatment of mandibular angle fractures with miniplate osteosynthesis according to Champy. Int J Oral Maxillofac Surg 2002;31(4):345-8.

Gibbs $\mathrm{CH}$, Anusavice KJ, Young HM, Jones JS, Esquivel-Upshaw JF. Maximum clenching force of patients with moderate loss of posterior tooth support: a pilot study. J Prosthet Dent 2002;88(5):498-502.

Gingrass DJ, Messer EJ. Rigid noncompressive pin fixation of the mandibular sagittal split osteotomy. J Oral Maxillofac Surg 1986;44(5):413-7.

Gomes PP, Guimaraes Filho R, Mazzonetto R. Evaluation of the bending strength of rigid internal fixation with absorbable and metallic screws in mandibular ramus sagittal split osteotomy: in vitro study. Pesqui Odontol Bras 2003;17(3):267-72.

Hagberg C. Electromyography and bite force studies of muscular function and dysfunction in masticatory muscles. Swed Dent J 1986;37 Suppl:1-64.

Hammer B, Ettlin D, Rahn B, Prein J. Stabilization of the short sagittal split osteotomy: in vitro testing of different plate and screw configurations. J Craniomaxillofac Surg 1995;23(5):321-4. 
Harada K, Watanabe M, Ohkura K, Enomoto S. Measure of bite force and occlusal contact area before and after bilateral sagittal split ramus osteotomy of the mandible using a new pressure-sensitive device: a preliminary report. J Oral Maxillofac Surg 2000;58(4):370-3.

Haug RH. Experimental analysis of functional stability of sagittal split ramus osteotomies secured by miniplates and position screws [discussion]. J Oral Maxillofac Surg 1996;54:1324-6.

Haug RH. The effects of screw number and length on two methods of tension band plating. J Oral Maxillofac Surg 1993;51(2):159-62.

Haug RH, Barber JE, Punjabi AP. An in vitro comparison of the effect of number and pattern of positional screws on load resistance. J Oral Maxillofac Surg 1999;57(3):300-8.

Hegtvedt AK, Michaels GC, Beals DW. Comparison of the resistance of miniplates and microplates to various in vitro forces. J Oral Maxillofac Surg 1994;52(3):251-7.

Hughes AN, Jordan BA. The mechanical properties of surgical bone screws and some aspects of insertion practice. Injury 1972;4:25-38.

Hunsuck EE. A modified intraoral sagittal splitting technic for correction of mandibular prognathism. J Oral Surg 1968;26:249-52.

Ikemura K, Kouno Y, Shibata H, Yamasaki K. Biomechanical study on monocortical osteosynthesis for the fracture of the mandible. Int J Oral Surg 1984;13(4):307-12.

Ikemura K, Hidaka H, Etoh T, Kabata K. Osteosynthesis in facial bone fractures using miniplates: clinical and experimental studies. J Oral Maxillofac Surg 1988;46(1):10-4.

Jeter TS, Van Sickels JE, Dolwick MF. Modified techniques for internal fixation of sagittal ramus osteotomies. J Oral Maxillofac Surg 1984;42(4):270-2.

Kempf KK. Transoral technique for rigid fixation of sagittal ramus osteotomies. J Oral Maxillofac Surg 1987;45:1077-9. 
Kim HC, Essaki S, Kameyama T, Yamamoto K. Photoelastic analysis of internal screw fixation after sagittal split ramus osteotomy. J Cranio Maxillofac Surg 1993;21:266-70.

Kim HC, Essaki S, Kameyama T. Comparison of screw placement patterns on the rigidity of sagittal split osteotomy: technical note. J Cranio Maxillofac Surg 1995;23:54-6.

Kohn DH. Functional stability of sagittal split ramus osteotomies: effects of positional screw size and placement configuration [discussion]. J Oral Maxillofac Surg 1996;54(5):609-10.

Kohn DH, Richmond EM, Dootz ER, Feinberg SE, Pietrzak WS. In vitro comparison of parameters affecting the fixation strength of sagittal split osteotomies. J Oral Maxillofac Surg 1995;53(12):1374-83.

Koolstra JH, van Eijden TM, Weijs WA, Naeije M. A three-dimensional mathematical model of the human masticatory system predicting maximum possible bite forces. $\mathrm{J}$ Biomech 1988;21(7):563-76.

Krekmanov L, Lilja J. Orthognathic surgery with no post-operative intermaxillary fixation. Scand J Plast Reconst Surg 1986;21:189-94.

Lee J, Piecuch JF. The sagittal ramus osteotomy. Stability of fixation with internal miniplates. Int J Oral Maxillofac Surg 1992;21(6):326-30.

Lindorf $\mathrm{HH}$. Sagittal ramus osteotomy with tandem screw fixation. Technique and results. J Maxillofac Surg 1986;14(6):311-6.

Loukota RA, Shelton JC. Mechanical analysis of maxillofacial miniplates. Br J Oral Maxillofac Surg 1995;33(3):174-9.

Maurer P, Holweg S, Schubert J. Finite-element-analysis of different screw-diameters in the sagittal split osteotomy of the mandible. J Craniomaxillofac Surg 1999;27(6):365-72.

Maurer P, Knoll WD, Schubert J. Comparative evaluation of two osteosynthesis methods on stability following sagittal split ramus osteotomy. J Craniomaxillofac Surg 2003;31(5):284-9. 
Mayo $\mathrm{KH}$, Ellis $\mathrm{E}$ 3rd. Stability of the mandible after advancement and use of dental plus skeletal maxillomandibular fixation: an experimental investigation in Macaca mulatta. J Oral Maxillofac Surg 1987;45(3):243-50.

McDonald WR. Champy bone plate fixation in sagittal split osteotomy for mandibular advancement. Int J Adult Orthod Orthog Surg 1987;2:89-92.

Mommaerts MY. Lag screw versus wire osteosynthesis in mandibular advancement. Int J Adult Orthodon Orthognath Surg 1991;6(3):153-60.

Muller JT, Bach DE. A simplified technique for percutaneous rigid fixation of sagittal ramus osteotomies. J Oral Maxillofac Surg 1989;47:93-5.

Murphy MT, Haug RH, Barber JE. An in vitro comparison of the mechanical characteristics of three sagittal ramus osteotomy fixation techniques. J Oral Maxillofac Surg 1997;55(5):489-94.

Niederdellmann $\mathrm{H}$, Shetty V, Collins FJ. Controlled osteosynthesis utilizing the position screw. Int J Adult Orthodon Orthognath Surg 1987;2(3):159-62.

Nissenbaum M, Lownie M, Cleaton-Jones P. Relative displacement resistance of standard and low-profile bone plates in experimental mandibular angle fractures. Oral Surg Oral Med Oral Pathol Oral Radiol Endod 1997;83(4):427-32.

Obeid G, Lindquist CC. Optimal placement of bicortical screws in sagittal split ramus osteotomy of mandible. Oral Surg Oral Med Oral Pathol 1991;71(6):665-9.

Ochs MW. Bicortical screw stabilization of sagittal split osteotomies. J Oral Maxillofac Surg 2003;61(12):1477-84.

Olson RA, Fonseca RJ, Zeitler DL, Osbon DB. Fractures of the mandible: a review of 580 cases. J Oral Maxillofac Surg 1982;40(1):23-8.

Ozden B, Alkan A, Arici S, Erdem E. In vitro comparison of biomechanical characteristics of sagittal split osteotomy fixation techniques. Int J Oral Maxillofac Surg 2006;35(9):837-41. 
Paulus GW, Steinhauser EW. A comparative study of wire osteosynthesis versus bone screws in the treatment of mandibular prognathism. Oral Surg Oral Med Oral Pathol 1982;54:2-6.

Perren SM. Biomechanical basis of fracture treatment. Orthopade 1992;21:3-10.

Perrott DH, Lu YF, Pogrel MA, Kaban LB. Stability of sagittal split osteotomies. A comparison of three stabilization techniques. Oral Surg Oral Med Oral Pathol $1994 ; 78(6): 696-704$.

Peterson GP, Haug RH, Van Sickels J. A biomechanical evaluation of bilateral sagittal ramus osteotomy fixation techniques. J Oral Maxillofac Surg 2005;63(9):1317-24.

Proffit WR, Turvey TA, Fields HW, Phillips C. The effect of orthognathic surgery on occlusal force. J Oral Maxillofac Surg 1989;47(5):457-63.

Putnam GD, Bouwman JP, Tuinzing DB. Stability of the osteotomy site following bilateral sagittal split osteotomy: screw fixation v IMF. Br J Oral Maxillofac Surg 1993;31(4):213-6.

Saka B. Mechanical and biomechanical measurements of five currently available osteosynthesis systems of self-tapping screws. Br J Oral Maxillofac Surg 2000;38:705.

Sheperd JP, Dohvoma CN, Harradine NWT. Screw fixation after mandibular sagittal split osteotomy: an intraoral approach. Br J Oral Maxillofac Surg 1991;29:325-9.

Schlicke LH, Panjabi MM, White AA 3rd. Optimal orientation of transfixation screws across oblique fractures lines. Clin Orthop Relat Res 1979;143:271-7.

Schwartz HC, Relle RJ. Bicortical-monocortical fixation of the sagittal mandibular osteotomy. J Oral Maxillofac Surg 1996;54(2):234-5.

Schwimmer A, Greenberg AM, Kummer F, Kaynar A. The effect of screw size and insertion technique on the stability of the mandibular sagittal split osteotomy. J Oral Maxillofac Surg 1994;52:45-8. 
Shetty $\mathrm{V}$. An in vitro comparison of the mechanical characteristics of three sagittal ramus osteotomy fixation techniques [discussion]. J Oral Maxillofac Surg 1997;55(5):494-95.

Shetty V, Freymiller E, McBrearty D, Caputo AA. Experimental analysis of functional stability of sagittal split ramus osteotomies secured by miniplates and position screws. J Oral Maxillofac Surg 1996a;54(11):1317-24.

Shetty V, Freymiller E, McBrearty D, Caputo AA. Functional stability of sagittal split ramus osteotomies: effects of positional screw size and placement configuration. $\mathrm{J}$ Oral Maxillofac Surg 1996b;54(5):601-9.

Shufford EL, Kraut RA. Passive rigid fixation of sagittal split osteotomy. Oral Surg Oral Med Oral Pathol 1989;68(2):150-3.

Smith BR, Rajchel JL, Waite DE, Read L. Mandibular anatomy as it relates to rigid fixation of the sagittal ramus split osteotomy. J Oral Maxillofac Surg 1991a;49(3):2226.

Smith BR, Rajchel JL, Waite DE, Read L. Mandibular ramus anatomy as it relates to the medial osteotomy of the sagittal split ramus osteotomy. J Oral Maxillofac Surg $1991 b ; 49(2): 112-6$.

Souyris F. Sagittal splitting and bicortical screw fixation of the ascending ramus.J Maxillofac Surg 1978;6(3):198-203.

Spiessl B. Rigid internal fixation after sagittal split osteotomy of the ascending ramus. In: Ipiessl B, editor. New concepts in maxillifacial bone surgery. New York: SpringerVerlag; 1976. p. 115-22.

Steinhauser EW. Bone screws and plates in orthognathic surgery. Int J Oral Surg 1982;11(4):209-16.

Stoelinga PJ, Borstlap WA. The fixation of sagittal split osteotomies with miniplates: the versatility of a technique. J Oral Maxillofac Surg 2003;61(12):1471-6.

Teltzrow T, Kramer FJ, Schulze A, Baethge C, Brachvogel P. Perioperative complications following sagittal split osteotomy of the mandible. J Craniomaxillofac Surg 2005;33(5):307-13. 
Tharanon W. Comparison between the rigidity of bicortical screws and a miniplate for fixation of a mandibular setback after a simulated bilateral sagittal split osteotomy. J Oral Maxillofac Surg 1998;56(9):1055-8.

Throckmorton GS, Ellis E 3rd, Winkler AJ, Dechow PC. Bone strain following application of a rigid bone plate: an in vitro study in human mandibles. J Oral Maxillofac Surg 1992;50(10):1066-73.

Throckmorton GS, Buschang PH, Ellis E 3rd. Improvement of maximum occlusal forces after orthognathic surgery. J Oral Maxillofac Surg 1996;54(9):1080-6.

Throckmorton GS, Throckmorton LS. Quantitative calculations of temporomandibular joint reaction forces--I. The importance of the magnitude of the jaw muscle forces. $J$ Biomech 1985;18(6):445-52.

Trauner $\mathrm{R}$, Obwegeser $\mathrm{H}$. The surgical correction of mandibular prognathism and retrognatia with consideration of genioplasty. Surgical procedures to correct mandibular prognathism and reshaping of the chin. Oral Surg Oral Med Oral Pathol 1957;10:677-89.

Tucker MR, Ochs MW. Use of rigid internal fixation for management of intraoperative complications of mandibular sagittal split osteotomy. Int J Adult Orthodon Orthognath Surg 1988;3(2):71-80.

Tulasne JF, Schendel SA. Transoral placement of rigid fixation following sagittal ramus split osteotomy. J Oral Maxillofac Surg 1989;47(6):651-2.

Turvey T. Intraoral self-threading screw fixation for sagittal osteotomies: early experience. Int J Adult Orthod Orthog Surg 1986;1:243-5.

Uckan S, Schwimmer A, Kummer F, Greenberg AM. Effect of the angle of the screw on the stability of the mandibular sagittal split ramus osteotomy: a study in sheep mandibles. Br J Oral Maxillofac Surg 2001;39:266-8.

Van Sickels JE. A comparative study of bicortical screws and suspension wires versus bicortical screws in large mandibular advancements. J Oral Maxillofac Surg 1991;49(12):1293-8.

Van Sickels JE, Peterson GP, Holms S, Haug RH. An in vitro comparison of an adjustable bone fixation system. J Oral Maxillofac Surg 2005;63(11):1620-5. 
Van Sickels JE, Richardson DA. Stability of orthognathic surgery: a review of rigid fixation. Br J Oral Maxillofac Surg 1996;34(4):279-85.

Watzke IM, Tucker MR, Turvey TA. Lag screw versus position screw techniques for rigid internal fixation of sagittal osteotomies: a comparison of stability. Int J Adult Orthod Orthognath Surg 1991;6:19-27.

Watzke IM, Turvey TA, Phillips C, Proffit WR. Stability of mandibular advancement after sagittal osteotomy with screw or wire fixation: a comparative study. J Oral Maxillofac Surg 1990;48(2):108-21.

Wittenberg JM, Wittenberg RH, Hipp JA. Biomechanical properties of resorbable poly-L-lactide plates and screws: a comparison with traditional systems. J Oral Maxillofac Surg 1991;49(5):512-6

Wolford LM, Bennett MA, Rafferty CG. Modification of the mandibular ramus sagittal osteotomy. Oral Surg Oral Med Oral Pathol 1987;64:146-55. 
ANEXO A - Parecer do comitê de ética em pesquisa

\section{PARECER}

O Grupo de Trabalho indicado pelo Comitê de Ética em Pesquisa APROVOU o protocolo de pesquisa "Avaliação da estabilidade na fixação da osteotomia sagital mandibular utilizando parafusos bicorticais inseridos com angulação de $60^{\circ}$ e $90^{\circ}$. Estudo biomecânico em mandíbulas de carneiro", de responsabilidade do pesquisador Higor Landgraf, sob a orientação do Professor Doutor Elio Hitoshi Shinohara.

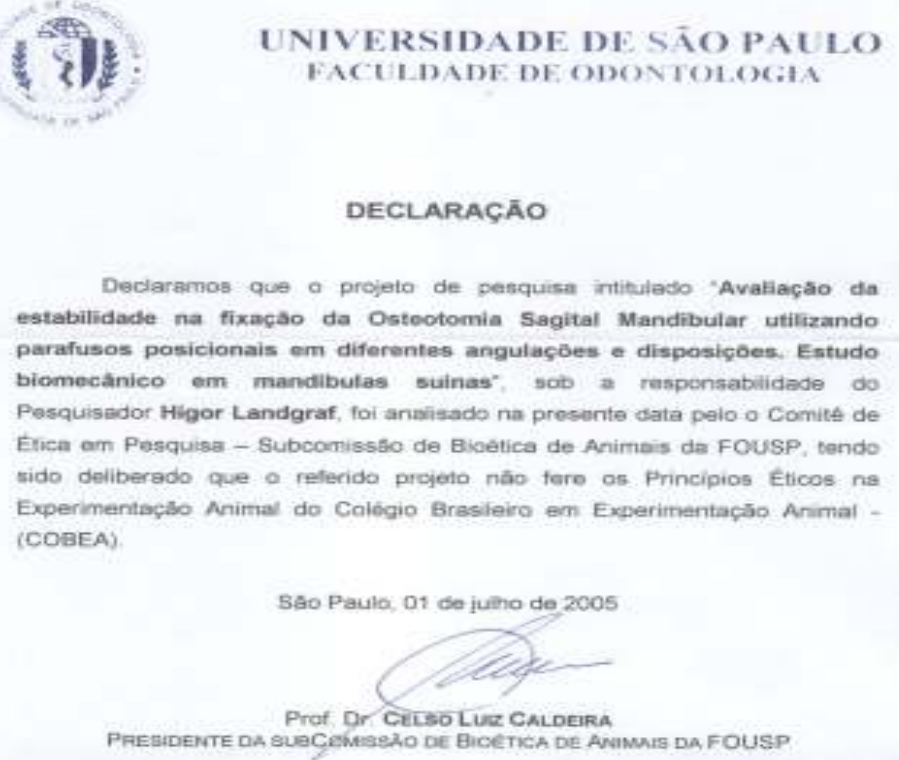


ANEXO B - Resultados individuais obtidos das mandíbulas e a estatística descritiva

\begin{tabular}{|c|c|c|c|c|c|}
\hline GRUPO & $\begin{array}{l}\text { NÚMERO DA } \\
\text { MANDÍBULA }\end{array}$ & $\begin{array}{l}\text { RIGIDEZ } \\
\text { INICIAL } \\
\text { (N/mm) }\end{array}$ & $\begin{array}{c}\text { RIGIDEZ APÓS } \\
\text { OSTEOTOMIA } \\
\text { (N/mm) }\end{array}$ & $\begin{array}{c}\text { DIFERENÇA } \\
\text { DE RIGIDEZ } \\
\text { (N/mm) }\end{array}$ & $\begin{array}{l}\text { RESISTÊNCIA A } \\
\text { FRATURA (N) }\end{array}$ \\
\hline \multirow{10}{*}{$60^{\circ}$} & I & 452 & 115 & 336 & 425 \\
\hline & II & 483 & 98 & 385 & 280 \\
\hline & III & 475 & 46 & 430 & 222 \\
\hline & IV & 649 & 126 & 523 & 266 \\
\hline & V & 523 & 169 & 354 & 195 \\
\hline & Média & 516 & 111 & 406 & 278 \\
\hline & DesvPad & 79 & 45 & 75 & 89 \\
\hline & Coef Var & 15 & 40 & 18 & 32 \\
\hline & Máximo & 649 & 169 & 523 & 425 \\
\hline & Mínimo & 452 & 46 & 336 & 195 \\
\hline \multirow{10}{*}{$90^{\circ}$} & VI & 576 & 61 & 515 & 505 \\
\hline & VII & 436 & 126 & 310 & 343 \\
\hline & VIII & 468 & 123 & 344 & 386 \\
\hline & IX & 506 & 133 & 913 & 363 \\
\hline & $x$ & 492 & 231 & 261 & 642 \\
\hline & Média & 495 & 135 & 469 & 448 \\
\hline & DesvPad & 52 & 61 & 266 & 125 \\
\hline & Coef Var & 11 & 45 & 57 & 28 \\
\hline & Máximo & 576 & 231 & 913 & 642 \\
\hline & Mínimo & 436 & 61 & 261 & 343 \\
\hline \multirow{3}{*}{ Hígida } & $X I$ & & & & 2344 \\
\hline & XII & & & & 2246 \\
\hline & Média & & & & 2295 \\
\hline
\end{tabular}

